\title{
Characterization and Degradation of Polyhydroxyalkanoates (PHA), Polylactides (PLA) and PHA-PLA Blends
}

\section{Rafeya Sohail}

Microbiology and Molecular Genetics, University of the Punjab, Lahore

Nazia Jamil ( $\square$ nazia.mmg@pu.edu.pk)

Microbiology and Molecular Genetics, University of the Punjab Lahore

\section{Research}

Keywords: Degradation, FT-IR, Mixed Culture, PHA-PLA blends, Polyhydroxyalkanoates, Polylactides, SEM, Soil

Posted Date: December 1st, 2020

DOI: https://doi.org/10.21203/rs.3.rs-113670/v1

License: (c) (i) This work is licensed under a Creative Commons Attribution 4.0 International License.

Read Full License 
1 Characterization and Degradation of Polyhydroxyalkanoates

2 (PHA), Polylactides (PLA) and PHA-PLA blends

3 Rafeya Sohail ${ }^{1}$, Nazia Jamil ${ }^{1 *}$

$4 \quad{ }^{1}$ Department of Microbiology and Molecular Genetics, University of the Punjab, Quaid-e-Azam

5 Campus, Lahore 54590, Punjab, Pakistan

$6 *$ Correspondence:

$7 \quad$ Nazia Jamil

$8 \quad$ nazia.mmg@pu.edu.pk 


\section{Abstract}

11 Biodegradable biopolymers such as polyhydroxyalkanoates (PHA) and polylactide (PLA) have

12 wide range of applications in almost all sectors. Degradation of these polymers, however

13 efficient, still creates a paradox with green chemistry principles. Blending of these polymers can

14 potentially decrease plastic pollution due to their increased biodegradability. During this study,

15 PHAwas produced using bacterial strains DL3; Bacillus subtilis (MT043898), PWA; Bacillus

16 subtilis (MH142143), PWC; Pseudomonas aeruginosa (MH142144), PWF; Bacillus tequilensis

17 (MH142145) and PWG; Bacillus safensis (MH142146). Corn-based PLA was produced

18 chemically and physically blendedwith PHA (PHA-PLA blends). During molecular studies,

19 PHA, PLA and PHA-PLA blendswere characterized via FT-IR analysis, SEM, and light

20 microscopy indicating successful blending of PHA-PLA samples. FTIR results indicated PHA

21 produced by strain PWF and mixed culture wasmcl-PHA. Degradation of polymers and

22 copolymer blends was studied by bacteria for 12 weeks and in different environmental systems

23 i.e. in soil, water, air, and heat for 20 weeks. Degradation analysis indicated highest degradation

24 of PLA, followed by PHA-PLA blends. Among these systems, degradation was favored in soil

25 (80\%), followed by water (78\%) and air (78\%). Least degradation (76\%) was observed on heat

26 exposure. Sample degradation observed using light microscope and SEM showed fungal and 27 bacterial colonization amidst cracks, crevices, bumps, and fractures after 20 weeks. 28 Understanding the role of ambient microbial population during degradation and its impact on 29 natural soil environment can be the focus of future studies.

\section{Keywords}


31 Degradation, FT-IR, Mixed Culture, PHA-PLA blends, Polyhydroxyalkanoates, Polylactides,

32 SEM, Soil.

\section{$33 \quad 1 \quad$ Introduction}

34 Traditional fossil fuel and petrochemical based plastics have been a commodity since first

35 production of plastics (Johnston et al 2017; Radecka et al 2016). The use of plastics has been

36 increasing since they can be easily manipulated both chemically and physically to suit the current

37 need (Reddy et al 2003). These properties have also made their use excessive and their eventual

38 disposal irresponsible (Fossi et al 2020). In Europe, China and USA, plastic wastes account for

$397 \%, 14 \%$ and $11.8 \%$ out of total generated municipal solid waste, respectively (Radecka et al

40 2016; Verma et al 2016; Johnston et al 2017).Biodegradable plastics are now being utilized as a

41 suitable replacement of synthetic nondegradable plastics, in order to manage and control

42 environmental pollution(Muniyasamy et al 2019).Biodegradable polymers can decompose in

43 natural environment - within almost a year or less - to water, carbon dioxide, methane, biomass

44 and inorganic compounds (Leja and Lewandowicz 2010). These degradation products can be

45 utilized to enrich soil and/or as microbial metabolic substrates. Nevertheless, even biodegradable

46 plastics are not a cure-all for the pollution caused by plastics (Narancic et al 2018). Many micro

47 and nano particles are released during the degradation of biodegradable plastics (Sintim et al

48 2019). Home composting of many classes of biodegradable plastics cannot be achieved easily

49 (Narancic et al 2018). Blending of different polymers - both synthetic and bio-based - to obtain

50 various copolymers with desirable chemical and physical properties has become very versatile

51 (Leja and Lewandowicz 2010). Recent studies have been conducted on utilization of 
52 biodegradable polymer blends such as PHA-PLA blends, PLA/ starch composites, PLA-PCL

53 blends, PHB-starch blends, PHB/P(HB-co-HV) blends, etc. (Muthuraj et al 2018; Shogren 2009).

54 Polyhydroxyalkanoates (PHA) have extensiveimport due to their biodegradability, 55 biocompatibility, structural diversity, and efficient bio-friendly production using 56 microorganisms( $\mathrm{Li}$ et al 2016). PHA are produced as carbon storage inclusion bodies by 57 incorporation of hydroxy acid (HA) subunits. A single PHA molecule contains $\sim 600$ to $35000 R$ -

58 hydroxy fatty acid subunits; where $R$ is the side chain alkyl group (Tan et al 2014). $R$ alkyl group

59 can be a saturated, non-saturated, branched, or substituted alkyl group. Most studied and best

60 characterized PHA is polyhydroxybutyrate, a homopolyester composed of $R$-3-hydroxybutyrate

61 monomers (Koller et al 2015). The spectrum of PHA potentials ranges from applications as

62 packaging films with various properties like UV resistance to high quality therapeutic or 63 pharmaceutical materials like thermo-sensitive adhesives, carrier materials for controlled release

64 of drugs in vivo, conduits guiding nerve repairs, implants, sutures, smart latexes, meshes, or a 65 tissue engineering scaffolds etc. (Koller et al 2015; Koller 2018). The monomeric and oligomeric 66 products of PHA degradation in vivo, do not cause toxicity or exert any negative effect on living 67 cells, making them highly significant for medical field applications (Koller 2018). PHAs have 68 been approved for clinical utilization in tissue engineering, biomedical devices, artificial organs 69 and repair patches by United States Foods and Drugs Administration (USFDA) (Shah and 70 Vasava 2019).

71 Polylactides (PLA), on the other hand, are aliphatic polyesters, produced from natural sources 72 such as potato starch, wheat, corn, rice bran and biomass etc. (Shah and Vasava 2019; Ausejo et 73 al 2018).Use of starchy source such as corn, wheat, beets, and potatoes etc. for PLA production 74 utilizes $65 \%$ less energy, reduces generation of greenhouse gases by $68 \%$ and contains no toxins 
75 (Muller et al 2017; Royte 2006; Rudnik 2019). Polylactides are highly transparent plastics with

76 low processing temperature high elasticity modulus and high strength ( $\mathrm{Su}$ et al 2019; Shah and

77 Vasava 2019)making them a popular material in many industrial sectors (Msuya et al 2017).

78 PLA, in fact, is also USFDA approved for applications in therapeutics and medical sectors (Shah

79 and Vasava 2019). Thermoplastic PLA composed of lactidemonomeric units - synthesizedeither

80 by direct polycondensation or ring-opening polymerization of lactides - can be grouped with

81 aliphatic polyester family made from $\alpha$-hydroxyl acids (Pretula et al 2016; Msuya et al

82 2017).Main constituent of PLA is a chiral monomer - existing in two enantiomeric forms i.e. L -

83 and D- configurations - termed as 2-hydroxypropionic acid (Shah and Vasava 2019; Zhang and

84 Thomas 2011; Kricheldorf 2001).

85 Blends of PHA-PLA show increased crystallinity, elasticity, thermal stability, and 86 biodegradability (Roy and Visakh 2014; Msuya et al 2017; Zhang and Thomas 2011; Koller et al

87 2015; Su et al 2019). Biodegradative ability of biopolymers is strongly influenced by

88 physiochemical nature of biopolymer i.e. stereoregularity, accessibility and crystallinity (Ray

89 and Kalia 2017a). Copolymer blends of biodegradable thermoplastics like PHA and PLA only

90 serve to enhance their potential for consumer applications (Rasal et al 2008).Biobased

91 biodegradable PHA-PLA copolymer blends being biocompatible have potential applications in

92 food, medicine, and agricultural sectors (Iwata 2015).Blending of PHA and high molecular

93 weight PLA conducted previously reports that even though these blends show a defined

94 interaction, these are not completely miscible(Zhang and Thomas 2011). Blending low molecular

95 weight PLA with PHA could serve to enhance miscibility as well as biodegradability.

96 Current study was aimed at the production of PHA using previously isolated PHA producers.

97 Corn based PLA having low molecular weight was produced chemically and biopolymers PHA 
98 and PLA were blended physically. Biopolymers PHA, PLA and PHA-PLA copolymer blends

99 were analyzed by light microscopy, scanning electron microscopy, and fourier transform infrared

100 spectroscopy. Current study also addressed the degradation of these copolymer blends by

101 bacteria and in different environmental systems i.e. soil, water, heat, and air.

\section{$102 \quad 2 \quad$ Results}

\section{$103 \quad$ 2.1 PHA, PLA and PHA-PLA Production}

104 Solvent cast films of PHA were obtained after evaporation of chloroform. After 24 hours, highest

105 biomass $(12.91 \mathrm{~g} / \mathrm{L})$ on glucose was by PWC while highest PHA production $(10.27 \mathrm{~g} / \mathrm{L} ; 84 \%)$

106 after $24 \mathrm{~h}$ was by strain PWF. PWC gave $7.73 \mathrm{~g} / \mathrm{L}(60 \%)$ PHA production while PWF gave

$107 \quad 12.30 \mathrm{~g} / \mathrm{L}$ biomass. Highest percentage PHA production (87\%; $9.28 \mathrm{~g} / \mathrm{L})$, however, was by

108 mixed culture. Lowest biomass $(5.07 \mathrm{~g} / \mathrm{L})$ and PHA production $(1.94 \mathrm{~g} / \mathrm{L} ; 27 \%)$ were by strain

109 PWG and DL3 respectively. Biomass and PHA production by strains DL3 and PWG were 7.10

$110 \mathrm{~g} / \mathrm{L}$ and $1.74 \mathrm{~g} / \mathrm{L}(34 \%)$ respectively. Biomass and PHA production by strains PWA were 11.20

$111 \mathrm{~g} / \mathrm{L}$ and $4.42 \mathrm{~g} / \mathrm{L}$ (39\%). Biomass increased exponentially till $96 \mathrm{~h}$, however \% PHA production

112 decreased after $48 \mathrm{~h}$ except in case of DL3. After $96 \mathrm{~h}$, DL3 gave 22\% (6.63 g/L) PHA

113 production (Table 1).PHA films of PWA, PWC, PWF, PWG, DL3 and mixed culture on glucose

114 were labelled as A, C, F, G, DL3 and M. Films thickness was approximately $1.5 \mathrm{~mm}$.

115 Transparent, thin films of corn based polylactide having $1.0 \mathrm{~mm}$ thickness were obtained. Films

116 had a smooth texture however surface was slightly ridged due to pasting on aluminum foil. PHA-

117 PLA blends (1:1) were obtained as solvent cast films with $1.5 \mathrm{~mm}$ film thickness. Blends were

118 labelled as PA, PC, PF, PG, PDL3 and PM for PHA-PLA blends using PHA sample of PWA,

119 PWC, PWF, PWG, DL3 and mixed culture, respectively. 


\begin{tabular}{|c|c|c|c|c|}
\hline Strains & Time (hours) & Biomass (g/L) & PHA (g/L) & \% РНA (\%) \\
\hline \multirow{4}{*}{ PWA } & 24 & 11.20 & 4.42 & 39 \\
\hline & 48 & 23.70 & 8.40 & 35 \\
\hline & 72 & 37.50 & 10.43 & 28 \\
\hline & 96 & 52.20 & 12.70 & 24 \\
\hline \multirow{4}{*}{ PWC } & 24 & 12.91 & 7.73 & 60 \\
\hline & 48 & 18.41 & 10.73 & 58 \\
\hline & 72 & 33.05 & 9.64 & 29 \\
\hline & 96 & 49.91 & 12.82 & 26 \\
\hline \multirow{4}{*}{ PWF } & 24 & 12.30 & 10.27 & 84 \\
\hline & 48 & 20.80 & 15.91 & 76 \\
\hline & 72 & 39.40 & 13.94 & 35 \\
\hline & 96 & 48.40 & 18.36 & 38 \\
\hline \multirow{4}{*}{ PWG } & 24 & 5.07 & 1.74 & 34 \\
\hline & 48 & 11.03 & 2.92 & 26 \\
\hline & 72 & 28.40 & 8.10 & 29 \\
\hline & 96 & 41.17 & 9.04 & 22 \\
\hline \multirow{4}{*}{ DL3 } & 24 & 7.10 & 1.94 & 27 \\
\hline & 48 & 13.87 & 2.72 & 20 \\
\hline & 72 & 28.37 & 6.23 & 22 \\
\hline & 96 & 37.70 & 10.51 & 28 \\
\hline \multirow{4}{*}{$\begin{array}{l}\text { Mixed } \\
\text { culture }\end{array}$} & 24 & 10.66 & 9.28 & 87 \\
\hline & 48 & 24.33 & 14.01 & 58 \\
\hline & 72 & 41.67 & 14.52 & 35 \\
\hline & 96 & 51.08 & 17.75 & 35 \\
\hline
\end{tabular}




\subsection{Characterization of PHA-PLA Blends}

\section{$121 \quad$ 2.2.1 Fourier Transform Infrared (FT-IR) Analysis}

122 FT-IR analysis of PHA, PLA and PHA-PLA gave a unique signature for each chemical complex

123 in the sample within the FT-IR spectrum. Each spectrum was found to be a representative of

124 sample's chemical composition, directly associated with concentration of chemical

125 complexes.PHA sample of PWF showed absorption peaks at 1232.677, 1376.73, 1438.467,

$1261720.398,2854.296$ and $2928.38 \mathrm{~cm}^{-1}$ corresponding to $\mathrm{C}-\mathrm{O}-\mathrm{C}, \mathrm{CH}_{3}, \mathrm{CO}-\mathrm{N}-, \mathrm{C}=\mathrm{O},-\mathrm{CH}_{2}$ and -

$127 \mathrm{CH}_{3}$ groups respectively (Figure 1).PHA sample of mixed culture showed absorption peaks at $1281232.677,1376.73,1438.467,1654.545,1722.456,2854.296$ and $2928.38 \mathrm{~cm}^{-1}$ corresponding to

$129 \mathrm{C}-\mathrm{O}-\mathrm{C}, \mathrm{CH}_{3},-\mathrm{CO}-\mathrm{N}-, \mathrm{C}=\mathrm{O}, \mathrm{C}=\mathrm{O},-\mathrm{CH}_{2}$ and $-\mathrm{CH}_{3}$ groups respectively (Figure 2). Absorption 130 peaks in the range $605.02 \mathrm{~cm}^{-1}$ to $1115.37 \mathrm{~cm}^{-1}$ correspond to stretching vibrations of C-O and C-

131 C. Absorptions at 1720.398 and $1722.456 \mathrm{~cm}^{-1}$ are known PHA marker bands corresponding to 132 crystalline $\mathrm{C}=\mathrm{O}$ group vibrations(Aljuraifani et al 2019).PLA sample shows peaks at 1232.677, $1331376.73,1438.467,1654.545,1745.092,2854.296,2928.38$ and $3259.701 \mathrm{~cm}^{-1}$ corresponding to

134 C-O-C, $\mathrm{CH}_{3},-\mathrm{CO}-\mathrm{N}-, \mathrm{C}=\mathrm{O}, \mathrm{C}=\mathrm{O},-\mathrm{CH}_{2}$ and $-\mathrm{CH}_{3}$ and $-\mathrm{OH}$ groups (Figure 3). Absorptions at $1351745.092 \mathrm{~cm}^{-1}$ correspond to amorphous $\mathrm{C}=\mathrm{O}$ group vibrations. Absorption peaks in the range $136605.02 \mathrm{~cm}^{-1}$ to $1016.599 \mathrm{~cm}^{-1}$ correspond to stretching vibrations of C-O and C-C.PHA-PLA 137 sample of strain PWF (PF) showed peaks at 1232.677, 1376.73, 1438.467, 1654.545, 1720.398, 138 1745.092, 2854.296, 2928.38 and $3259.701 \mathrm{~cm}^{-1}$ corresponding to $\mathrm{C}-\mathrm{O}-\mathrm{C}, \mathrm{CH}_{3},-\mathrm{CO}-\mathrm{N}-, \mathrm{C}=\mathrm{O}$, $139 \mathrm{C}=\mathrm{O}, \mathrm{C}=\mathrm{O},-\mathrm{CH}_{2}$ and $-\mathrm{CH}_{3}$ and $-\mathrm{OH}$ groups (Figure 4). PHA-PLA sample of mixed culture 140 (PM) showed peaks at 1232.677, 1376.73, 1438.467, 1654.545, 1722.456, 1745.092, 2854.296, 1412928.38 and $3259.701 \mathrm{~cm}^{-1}$ corresponding to $\mathrm{C}-\mathrm{O}-\mathrm{C}, \mathrm{CH}_{3},-\mathrm{CO}-\mathrm{N}-, \mathrm{C}=\mathrm{O}, \mathrm{C}=\mathrm{O}, \mathrm{C}=\mathrm{O},-\mathrm{CH}_{2}$ and - 
$142 \mathrm{CH}_{3}$ and $-\mathrm{OH}$ groups (Figure 5). Absorption peaks in the range $605.02 \mathrm{~cm}^{-1}$ to $1016.599 \mathrm{~cm}^{-1}$

143 correspond to stretching vibrations of C-O and C-C.Absorptions at 1720.398 and $1722.456 \mathrm{~cm}^{-1}$

144 correspond to crystalline $\mathrm{C}=\mathrm{O}$ group vibrations while absorptions at $1745.092 \mathrm{~cm}^{-1}$ correspond to

145 amorphous $\mathrm{C}=\mathrm{O}$ group vibrations. Percentage crystallization values calculated for samples PLA,

146 F, M, PF and PM were 21.8\%, 28.8\%, 27.7\%, 33.7\% and 25.2\%, respectively. The presence of

147 both chemical complexes in PHA-PLA blendsas indicated by characterization results and the 148 increase in crystallization concluded the successful physical blending of these polymers.

\section{$149 \quad$ 2.2.2 Microscopy and Elasticity}

150 Light microscopy of PHA and PHA-PLA samples showed dark particlesagainst a light film

151 surface. Darker sites contained high concentration of particleswhile lighter areas contained low

152 concentration of particles. Micrographs of PLA samples showed no discernible small or large

153 particles, rather a smooth surface was observed. In PHA-PLA blends, the presence and size of

154 particles were significantly reduced. Samples of all PHA and PHA-PLA films showed surface

155 irregularities, varying in appearance and number with the variance in composition. Presence of

156 folds was also observed in all PHA samples indicating formation of folds during solvent casting

157 of PHA films. PHA-PLA samples showed red spots under natural light indicting a probable light

158 ionizing capability. These red spots disappeared on using in-built light bulb of microscope.

159 Crystal violet stained PLA samples showed that whole sample has been stained evenly. Surface

160 irregularities in PLA films appeared darker in contrast to smooth film surface. Crystal violet

161 stained PHA samples showed blue stained particlesagainst a light background. Almost all

162 particleshad been stained blue. In case of PHA-PLA blends, particlesas well as certain portions

163 of film (those containing PLA phase) had been stained blue. Due to increased uniform staining,

164 PHA-PLA blends appeared as brown or dark brown. On safranin stained PLA micrographs, spots 
165 of red color were distributed homogenously across the film. On safranin staining, PHA and

166 PHA-PLA samples appeared red against a light background. In case of PHA samples, film

167 margins were observed as a darker red in contrast to the lighter red dye attained by film surface.

168 In case of PHA-PLA samples, both margins and interior of film surface attained a uniform red

169 coloration. Scanning electron microscopy of PLA samples showed typical regular fracture

170 surfaces while those of PHA samples showed irregular fracture surfaces. PHA-PLA blends

171 showed both fracture surfaces i.e. typical regular and irregular (Figure 6). Tensile strength

172 measurement showed that low molecular weight PLA had lowest elasticity. PHA-PLA blends

173 showed highest elasticity followed closely by PHA samples.

\section{$174 \quad 2.3$ Degradative Analysis}

\section{$175 \quad$ 2.3.1 Bacterial Degradation}

176 During bacterial degradation only Bacillus subtilis degraded PHA, PLA and PHA-PLA films,

177 while Pseudomonas aeruginosa had no effect.At week 2, PHA sample F, G, DL3, PLA sample,

178 PHA-PLA sample PA and PDL3 were degraded. Highest degradation was of PHA sample G.

179 bacterial growth surrounding degraded polymer changed color to transparent.At week 4, 180 degradation rates followed the trend $\mathrm{G}>\mathrm{PDL} 3>\mathrm{DL} 3>\mathrm{PLA}>\mathrm{F}>\mathrm{PA}>\mathrm{C}>\mathrm{PG}>\mathrm{PM}$. Bacterial growth

181 surrounding degraded samples was milky in color,raised, bulbous and irregular (not in circular

182 form). Bacterial growth around PHA samples had a lustrous sheen while growth surrounding

183 PLA and PHA-PLA samples had no luster.Polymer color had changed to brown and edges had

184 become irregular.At week 8, bacterial growth surrounding polymer had changed color to light

185 brown and polymer edges had turned black. Degradation rates followed the pattern $186 \mathrm{G}>\mathrm{PDL} 3>\mathrm{PLA}>\mathrm{DL} 3>\mathrm{PC}>\mathrm{PA}>\mathrm{C}>\mathrm{F}>\mathrm{PM}$. At week 12, bacterial growth surrounding samples had 
187 turned dark brown. Degradation rates followed the same trend as at week 8. Overall highest

188 bacterial degradation was of PHA sample G.

\subsubsection{Degradation in Different Environmental Systems}

190 Morphologically all PHA, PLA, and PHA-PLA films were transparent and smooth initially but

191 gradually became rough in texture. Change in color of films also took place with time. At the end

192 of this study, all films had become rough and porous. Bacterial colonization of films was

193 observed in all cases. However, fungal penetration of films occurred only in case of water and

194 soil. As degradation prolonged, films become more prone to disintegration and fragmentation.

195 Molecular weight of films increased slightly at week 2 due to water uptake and then decreased 196 gradually. Percentage degradation followed the trend Soil $>$ Water $>$ Air $>$ Heat.

$197 \quad$ 2.3.2.1 Morphological Analysis of Samples

198 Soil degradation rates by PHA, PLA and PHA-PLA samples followed the trend $199 \mathrm{PLA}>\mathrm{PF}>\mathrm{PC}>\mathrm{PM}>\mathrm{PA}>\mathrm{PG}>\mathrm{F}>\mathrm{C}>\mathrm{M}>\mathrm{A}>\mathrm{G}>\mathrm{PDL} 3>\mathrm{DL} 3$ (Figure 7). Highest degradation rate of 200 approximately $80 \%$ by PLA (0.0202 g) at week 20 was followed by degradation rates of PF, PC, 201 PM, PA, PG, F, C, M, A, G, PDL3 i.e. 68\% (0.0316 g), 66\% (0.0345 g), 66\% (0.0345 g), 65\% $202(0.0345 \mathrm{~g}), 65 \%(0.0354 \mathrm{~g}), 61 \%(0.0391 \mathrm{~g}), 59 \%(0.0409 \mathrm{~g}), 55 \%(0.0450 \mathrm{~g}), 53 \%(0.0466 \mathrm{~g})$ $20349 \%$ (0.0512 g) and 46\% (0.0536 g) respectively. Lowest degradation rate i.e. 31\% was of PHA 204 sample DL3 whose weight decreased to $0.0687 \mathrm{~g}$.

205 Water degradation rates by PHA, PLA and PHA-PLA samples followed the trend 206 PLA $>$ DL3 $>$ PDL3 $>$ M $>$ G $>$ A $>$ PG $>$ PM $>$ C $>$ PA $>$ PC $>$ F $>$ PF (Figure 8). Highest degradation rate of 207 approximately $78 \%$ by PLA $(0.0217 \mathrm{~g})$ at week 20 was followed by degradation rates of DL3, 
208 PDL3, M, G, A, PG, PM, C, PA, PC, F i.e. 66\% (0.0340 g), 63\% (0.0372 g), 63\% (0.0371 g),

$20963 \%(0.0371 \mathrm{~g}), 62 \%(0.0381 \mathrm{~g}), 58 \%(0.0421 \mathrm{~g}), 56 \%(0.0440 \mathrm{~g}), 52 \%(0.0485 \mathrm{~g}), 50 \%(0.0501$

$210 \mathrm{~g}), 45 \%(0.0552 \mathrm{~g})$ and $42 \%(0.0577 \mathrm{~g})$ respectively. Lowest degradation rate i.e. $26 \%$ was of

211 PHA-PLA sample PF whose weight decreased to $0.0739 \mathrm{~g}$.

212 Air degradation rates by PHA, PLA and PHA-PLA samples followed the trend 213 PLA $>$ DL3 $>$ PDL3 $>$ G $>$ M $>$ A $>$ PG $>$ PM $>$ C $>$ PA $>$ PC $>$ F $>$ PF (Figure 9). Highest degradation rate of 214 approximately $78 \%$ by PLA $(0.0221 \mathrm{~g})$ at week 20 was followed by degradation rates of DL3, 215 PDL3, G, M, A, PG, PM, C, PA, PC, F i.e. 65\% (0.0347 g), 62\% (0.0379 g), 62\% (0.0379 g), $21662 \%(0.0378 \mathrm{~g}), 61 \%(0.0389 \mathrm{~g}), 57 \%(0.0429 \mathrm{~g}), 55 \%(0.0449 \mathrm{~g}), 51 \%(0.0494 \mathrm{~g}), 49 \%(0.0511$

$217 \mathrm{~g}), 44 \%(0.0562 \mathrm{~g})$ and $41 \%(0.0588 \mathrm{~g})$ respectively. Lowest degradation rate i.e. $25 \%$ was of 218 PHA-PLA sample PF whose weight decreased to $0.0754 \mathrm{~g}$.

219 Heat degradation rates by PHA, PLA and PHA-PLA samples in heat followed the trend 220 PLA $>$ DL3 $>$ M $>$ PDL3 $>$ G $>$ A $>$ PG $>$ PM $>$ C $>$ PA $>$ PC $>$ F $>$ PF $($ Figure 10). Highest degradation rate of 221 approximately $76 \%$ by PLA $(0.0241 \mathrm{~g})$ at week 20 was followed by degradation rates of DL3, 222 M, PDL3, G, A, PG, PM, C, PA, PC, F i.e. 62\% (0.0378 g), 59\% (0.0413 g), $59 \%$ (0.0413 g), $22359 \%(0.0413 \mathrm{~g}), 58 \%(0.0424 \mathrm{~g}), 53 \%(0.0468 \mathrm{~g}), 51 \%(0.0489 \mathrm{~g}), 46 \%(0.0539 \mathrm{~g}), 44 \%(0.0557$ $224 \mathrm{~g}), 39 \%(0.0613 \mathrm{~g})$ and 36\% (0.0641 g) respectively. Lowest degradation rate i.e. $18 \%$ was of 225 PHA-PLA sample PF whose weight decreased to $0.0821 \mathrm{~g}$.

226 Highest degradation measured among all polymer and copolymer films under soil after 20 weeks 227 (80\%) was of PLA whose weight decreased from $0.1000 \mathrm{~g}$ to $0.0202 \mathrm{~g}$. Transparent, smooth 228 PLA sample at week 0 changed morphologically to slightly yellow, rough sample at week 10. At 229 week 12, sample started to disintegrate slightly. At week 16, sample coloration changed to brown 
230 and at week 20, sample disintegrated completely to powdered from on application of slightest

231 pressure. Weight measurement showed a slight weight increase from week $2(0.1363 \mathrm{~g})$ to week

$2323(0.1279 \mathrm{~g})$, followed by a gradual decrease in weight up to week 20 (0.0202 g). Approximately

$23379-80 \%$ of PLA sample was completely degraded by week 12. Degradation rates decreased

234 after week 12 and remained almost same i.e. at $80 \%$ up to week 20. Degradation by weight

235 measured for PHA sample $\mathrm{F}$ was $61 \%$ with weight decrease from $0.1000 \mathrm{~g}$ to $0.0391 \mathrm{~g}$.

236 Transparent, smooth PHA sample F at week 0 changed to slightly yellow, slightly rough sample

237 at week 5 with $0.0781 \mathrm{~g}$ weight and $22 \%$ degradation. At week 8 , sample turned yellow and

238 rough. Weight dropped from $0.0747 \mathrm{~g}$ at week 6 to 0.0664 at week 8 with $34 \%$ degradation. At

239 week 10, $0.0584 \mathrm{~g}$ weight was measured with $42 \%$ degradation. At week 11, sample had started

240 to lose its elasticity. Elasticity loss occurred gradually from week 9 to week 12, during which

241 degradation rates slowed down. Degradation of $40 \%$ at week 9 was followed by $42 \%$ at week 10

242 and 11. At week 12, 44\% degradation by weight of sample F has occurred. At week 16, sample 243 coloration changed to brown and sample lost all elasticity turning brittle. At week 20, sample

244 disintegrated on application of pulling force, having no noticeable elasticity. Weight

245 measurement showed a gradual decrease in weight up to week 20 i.e. $0.0391 \mathrm{~g}$. Approximately

$24661 \%$ of PHA sample $\mathrm{F}$ had been degraded completely by week 20. Among all PHA-PLA

247 copolymer samples, highest degradation was by PHA-PLA sample PF (PLA blended with PHA

248 of strain PWF). At week 2, 26\% of sample had been degraded and weight had been reduced to

$2490.0737 \mathrm{~g}$. By the $10^{\text {th }}$ week, $51 \%$ of sample had been degraded. Weight of degraded sample was

250 recorded at 0.0503 g. During degradation, sample retained its initial morphology for 11 weeks.

251 At week 12, transparent, smooth sample changed morphologically to a slightly yellow, slightly

252 rough sample with $0.0429 \mathrm{~g}$ weight and 57\% degradation. Sample turned yellow at week 16 with 
$2530.0347 \mathrm{~g}$ weight and $65 \%$ degradation. At week 20 , sample weight was recorded at $0.0316 \mathrm{~g}$.

254 Sample PF showed a maximum degradation rate of $68 \%$ after 20 weeks soil burial.

255 In case of air, water and heat, highest degradation was also by PLA. For PHA andPHA-PLA 256 samples, highest degradation was achieved at week 20 by sample DL3 and PDL3, respectively.

257 In air, degradation of DL3at week 20 was $65 \%$, when sample weight had dropped down to 2580.0347 g. Initially, sample started as transparent and smooth. During week 2, sample became 259 cream colored and sample weight decreased to $0.0809 \mathrm{~g}$. At week 3, sample turned slightly 260 rough. Up to $22 \%$ of sample had been degraded and sample weight decreased to $0.0782 \mathrm{~g}$.

261 Sample changed color to yellow at week 6. Sample weight of $0.0722 \mathrm{~g}$ was recorded. A 262 degradation rate of $28 \%$ was measured.At week 9, sample become rough. Sample had degraded 263 up to $45 \%$ with an average weight of $0.9552 \mathrm{~g}$. At week 16, sample turned brownish.PHA-PLA 264 sample DL3 showed 62\% degradation with $0.0379 \mathrm{~g}$ weight at week 20. Sample changed color 265 to cream with 25\% degradation and $0.0753 \mathrm{~g}$ weight at week 7 . Sample showed $34 \%$ degradation 266 with $0.0659 \mathrm{~g}$ weight at week 9 . Sample had turned slightly rough with yellow coloration. At

267 week 12, sample coloration had changed to brownish yellow with $0.0550 \mathrm{~g}$ weight and $45 \%$ 268 degradation.

\section{$269 \quad$ 2.3.2.2 Microscopic Analysis of Samples}

\section{$270 \quad$ 2.3.2.2.1 Light Microscopy}

271 Air degraded PHA and PHA-PLA samples showed minimal bacterial colonization.

272 Samplesurface was riddled with cracks, crevices, and bumps. Heat degraded PHA and PHA-PLA

273 samples were observed as burnt with dark brown coloration against a light background except in 
274 case of PLA sample. PLA sample showed more pronounced surface roughening as compared to

275 other samples. No bacterial colonization was observed.Soil degraded PHA and PHA-PLA

276 samples showed bursting and swelling due to bacterial colonization. PLA sample showed

277 completely degraded surface morphology. Fungal hyphae were observed penetrating and

278 colonizing the PLA sample. Fungal hyphae were absent in case of PHA and PHA-PLA samples.

279 All water degraded PHA and PHA-PLA samples showed bacterial colonization causing swelling

280 in polymers. PLA sample of water also showed presence of fungi penetrating sample. Bacterial

281 and fungal colonization of sample was also observed.

\section{$282 \quad$ 2.3.2.2.2 Scanning Electron Microscopy}

283 SEM micrographs of polymer films before degradation showed typical fracture surfaces.

284 Distinctive features of degraded polymer films were observed on SEM microscopy after week 20

285 of soil burial (Figure 6). PHA sample of strain PWF before degradation showed typical fracture

286 surfaces and light reflecting particlesat $500 \mathrm{X}$ and $1000 \mathrm{X}$. At 2000X and $3000 \mathrm{X}$, irregular

287 surface morphology was observed. SEM micrographs of sample after degradation showed

288 presence of degradedparticles, crevices, cracks, bursts, and bacterial colonization. At $500 \mathrm{X}$,

289 bacterial colonization of sample surface was apparent while at $1000 \mathrm{X}$, sample showed irregular

290 fracture surfaces riddled with bumps and swells. At 2000 X, cracks and crevices were observed

291 between bumps and swells of sample. At $3000 \mathrm{X}$, small pieces of samples were observed to be

292 breaking down and off.

293 SEM micrographs of PLA sample before degradation showed an almost smooth surface having 294 regular fracture surfaces. PLA sample after degradation showed both bacterial and fungal 295 colonization of polymer surfaces. At 500 X, a webbed colonization pattern was observed 
296 encompassing, the sample. Fungal hyphae were further observed to penetrate the polymer.

297 Presence of pits, depressions, bumps, cracks, crevices, and swells was also apparent at $500 \mathrm{X}$,

298 indicating highest degradation in PLA sample. At 1000 X, polymer was observed to be breaking

299 off into small pieces. At $2000 \mathrm{X}$, these small pieces of sample were seen to be held together due

300 fungal penetration and webbed bacterial colonization. SEM micrograph of PLA sample at 3000

301 X, showed these small pieces as broken off small budding spheroids with irregular surfaces.

302 PHA-PLA sample before degradation showed a smooth surface riddled irregularly with a rough

303 surface at $500 \mathrm{X}$ i.e. regular fracture surfaces of PLA are more apparent. At $1000 \mathrm{X}$ and $2000 \mathrm{X}$,

304 small depressions and pits were observed indicating overlay of both polymeric fracture

305 surfaces.At $3000 \mathrm{X}$, at the site of pits and depressions, irregular fracture surfaces typical of PLA

306 were observed. After degradation, SEM micrographs of PHA-PLA sample at $500 \mathrm{X}$ showed a

307 branching colonization of sample. Sample surface was observed to be irregular having pits,

308 depressions, cracks, and cervices. No bumps or swells were observed. At $1000 \mathrm{X}$, numerous

309 small broken pieces of sample were observed. Large pieces were also seen to be breaking down.

310 At $2000 \mathrm{X}$ and $3000 \mathrm{X}$, sample surface was observed as being comprised of broken fragments

311 adhering to each other.

\section{Discussion}

313 Polyhydroxyalkanoate (PHA) was produced using previously isolated PHA producers (Sohail

314 and Jamil 2019; Sohail et al 2020).Polylactide (PLA) was produced using corn starch and solvent

315 cast PHA-PLA films were obtained after solvent induced recrystallization. PLA-PHA blends

316 showed improved elasticity since blending with PHA enhances crystallinity of PLA. This

317 property is attributed to the crystalline behavior of PHA which acts as filler in copolymer blends 
318 to enhance PLA matrix. There might also be strong interfacial bonding between these polymers,

319 however no definitive tests were performed to ascertain this bonding. Highly crystalline property

320 of PHA also improves mechanical properties since in PLA-PHA blends, PHA acts as filler as

321 well as nucleating agent. Similar findings were reported by Zhang et al. who studied effect of

322 PLA-PHB blends on thermal, mechanical and biodegradation properties (Zhang and Thomas 323 2011).

324 In both PHA samples, stretching vibrations of crystalline carbonyl group $(\mathrm{C}=\mathrm{O})$ for were found 325 at $1720.398 \mathrm{~cm}^{-1}$ and $1722.456 \mathrm{~cm}^{-1}$, while in PLA sample, stretching vibrations of amorphous 326 carbonyl group $(\mathrm{C}=\mathrm{O})$ for were found at $1745.092 \mathrm{~cm}^{-1}$ (Figure 1-3). FT-IR analysis of PHA 327 polymer confirms the presence of PHA functional group. Munir et al.have reported production of 328 mcl PHA by Bacillus subtilis, Pseudomonas aeruginosa and co-cultures on glucose(Munir and 329 Jamil 2018). Masood et al.also reported production of mcl and lcl PHA by Bacillus sp. on 330 glucose and olive oil, respectively (Masood et al 2017). Results of characterization and literature 331 review indicate that mcl PHA is produced.In case of PHA-PLA blends, both absorption bands 332 were observed due to blending of both polymers (Figure 4, 5).Zhang et al. reported similar 333 results for FT-IR spectrum of PLA showing strong absorption bands at $1745 \mathrm{~cm}^{-1}$ related to 334 vibration of amorphous carbonyl groups, and weak absorption bands at $1755 \mathrm{~cm}^{-1 \text { (Zhang and Thomas }}$ 335 2011). The absorption spectra of corn based PLA are very similar to those reported by Basnet $e t$ 336 al., who found FT-IR, TGA and DSC test results of corn based PLA similar to those of reference 337 PLA (Basnet and Wiberg 2016). The compositional analysis of the polymer is just a 338 confirmation of the PLA functional group, and notan exploration of potential new physical or 339 chemical characteristics. However, the process and/or polymer can be physically or chemically 340 modified.Zhang et al. also reported FT-IR spectra of PHA showing strong absorption bands at 
$3411718 \mathrm{~cm}^{-1}$ related to crystalline carbonyl stretching vibrations and weak absorption bands at 1740 $342 \mathrm{~cm}^{-1}$. They reported two strong bands at $1722 \mathrm{~cm}^{-1}$ for crystalline carbonyl and $1745 \mathrm{~cm}^{-1}$ for 343 amorphous carbonyl in PHA-PLA blends(Zhang and Thomas 2011).Absorption bands were also 344 observed at $3259.701 \mathrm{~cm}^{-1}$ assigned to stretching vibrations of -OH group. Absorption bands 345 corresponding to lateral monomeric units of aliphatic asymmetric alkyl groups were observed at 3462854.296 and $2928.38 \mathrm{~cm}^{-1}$. Absorption bands corresponding to stretching vibration of carbonyl 347 ester groups were observed at $1654.545 \mathrm{~cm}^{-1}$. Absorption bands corresponding to intracellular 348 amide groups were observed at $1438.467 \mathrm{~cm}^{-1}$. Absorption bands corresponding to stretching 349 vibrations of terminal alkyl groups were observed at $1376.73 \mathrm{~cm}^{-1}$. Absorption bands 350 corresponding to asymmetric C-O-C groups were observed at $1232.677 \mathrm{~cm}^{-1}$. Ismail et al. also 351 reported presence of $\mathrm{C}=\mathrm{O}, \mathrm{C}-\mathrm{H}, \mathrm{C}-\mathrm{O}$, and $\mathrm{O}-\mathrm{H}$ absorption bands indicating bioplastic formation 352 (Ismail et al 2016).

353 Aljuraifani et al. also reported similar absorption bands i.e. strong absorption band at 3456.43 $354 \mathrm{~cm}^{-1}$ corresponding to stretching vibrations of hydroxyl group, bands at 2986.44 and $2858.50 \mathrm{~cm}^{-}$

$355{ }^{1}$ corresponding to aliphatic alkyl groups, bands at $1637.56 \mathrm{~cm}^{-1}$ corresponding to $\mathrm{C}=\mathrm{O}$ and at $3561261.44 \mathrm{~cm}^{-1}$ corresponding to stretching vibrations of C-O group (Aljuraifani et al 2019). 357 Percentage crystallization values calculated for samples PHA, M, PM were 21.8\%, 27.7\% and $35825.2 \%$, respectively.Zhang et al. reported that the typical degree of crystallinity for PHA is about $35910.2 \%$ which can be increased up to $60 \%$ under high pressure crystallization (Zhang et al 2012). 360 Gopi et al. reported $26.4 \%$ degree of crystallization in poly-3-hydroxydodecanoate (P3HDD) 361 (Gopi et al 2018) while Yang also reported 22.75\% degree of crystallinity for PHA (Yang 2014). 362 Defined increase in crystallization was observed in case of sample PF i.e. $33.7 \%$ where samples $363 \mathrm{~F}$ and PLA had $28.8 \%$ and $21.8 \%$ crystallization respectively.Rasal et al. reported $20 \%$ increase 
364 in crystallinity of PLA PHA-g-PAAmand PAAm after solvent induced crystallization (Rasal et al 365 2008). Comparatively, values of \%crystallinity reported by Nerker et al. - on DSC of PHO, PLA 366 and PLA/PHO blends - were $24 \%$ for PLA, PHO (Polyhydroxy octanoate) and $16 \%$ for 367 PLA/PHO blend (Nerkar et al 2015). FT-IR results indicated that in PHA-PLA blends, particles 368 of PHA and PLA are distributed uniformly across the dried films of copolymer. These results 369 also account for the fact that in PHA-PLA blends, crystallizationof PLA is increased. In light 370 microscopy, absence of aggregates in PLA samples indicates that PLA is amorphous while in 371 contrast presence of aggregates andparticles in PHA samples is due to its crystallinity. In case of 372 PHA-PLA blends, PLA being amorphous reduces the presence and size of particles in crystalline 373 PHA significantly. In a study by Zhang et al., similar findings were reported i.e. crystalline PHB 374 dispersed in continuous phase of amorphous PLA. Zhang et al. reported lowering in rate of 375 crystallization due to reduction in size and number of PHB spherulites, to the extent that 376 crystalline phase of PHB became continuous (Zhang and Thomas 2011). In scanning electron 377 microscopy, presence of typical fracture surfaces in PLA samples are due to its amorphous 378 nature while PHA samples show the irregular fracture surfaces indicative of a crystalline 379 polymer. In PHA-PLA blends, both types of fracture surfaces were observed which showed that 380 PHA particles are distributes across amorphous PLA as fillers. Zhang et al. reported presence of 381 two phases in PHA-PLA blends using high molecular weight PLA, accounting for the fact that 382 those PHA-PLA blends were not miscible (Zhang and Thomas 2011).Overall, characterization 383 results indicated that although PHA and low molecular weight PLA polyesters were miscible, no 384 discernible strong molecular interactions were found between these two polymers. However, 385 there was a defined interaction between PHA and PLA particles. This interaction resulted in 386 increased elasticity, crystallinity, changes in mechanical properties. 
387 Many microbes have the ability to degrade PHA, however enzymatic degradation of biopolymers

388 also takes place in natural ecosystems. The effects of bacterial degradation by Bacillus subtilis 389 and Pseudomonas aeruginosa on PHA, PLA and PHA-PLA blendswithout the interfering effects

390 of enzymatic degradation were studied in aseptic conditions. Blends of PLA-PHA also showed

391 increased biodegradability in differentenvironmental systems i.e. air, water, soil, and heat.

392 Highest degradation was observed in soil (Figure 7), followed by degradation in water (Figure

393 8) and then air (Figure 9). Lowest degradation rates were observed on application of heat

394 (Figure 10).Following the overall degradation pattern, highest degradation was of PLA, 395 followed by PHA-PLA blends and lowest degradation was of PHA samples. Discoloration of 396 samples occurring with increasing time also accounts for their degradation. There was an initial

397 increase in weight at the beginning of experiment attributed to the absorption of water by PHA, 398 PLA and PHA-PLA blends. Increase in weight was especially defined in case of corn-based PLA 399 and PHA-PLA blends. This can be attributed to the fact that the building blocks of PLA are 400 hydrophilic. However, Zhang et al. reported increased water absorption by PHB compared to 401 synthetic PLA and lowered PLA degradation rate at room temperature (Zhang and Thomas 402 2011).Since, this study was conducted in uncontrolled environments, the richness of microbial 403 flora and other environmental properties could have affected the trend of degradation. 404 Differences from consistent degradative behavior can also be due to dissimilarity of PLA class 405 i.e. PLA synthesized by direct polymerization is more amorphous and has a more easily 406 degradative chemical nature as compared to PLA synthesized by ring 407 polymerization.Biodegradation patterns of polymer understudy could be due to their physical 408 form. Volova et al. reported high degradation of PHA polymer films compared to compacted 409 pellets of same weight and chemical composition in seawater. However, unchanged degree of 
410 crystallization was reported i.e. both amorphous and crystalline phases disintegrated equally

411 (Volova et al 2010).

412 Lowest degradation of polymer sample at $37^{\circ} \mathrm{C}$ indicates that these biopolymers are

413 biocompatible. However, polymer degradation has been reported to take place at high

414 temperatures. Sarasa et al. reported $63.6 \%$ biodisintegration of pieces made of PLA with

415 foaming agent and $79.7 \%$ biodisintegration degree in pieces made of PLA-corn(Sarasa et al

416 2009). Slow degradation at in heat and in water also indicates the probable applications of these

417 biopolymers as medical implant devices, drug carriers, tissue engineering etc. (Modjarrad and

418 Ebnesajjad 2013; Zinn et al 2001). Water soluble and/or biodegradable also have significance in

419 therapeutics and medical physiology ( $\mathrm{Li}$ and Loh 2015). Results indicate that air degraded

420 polymer samples had become a site for microbial colonization. Degradation in air was mainly

421 due to microbial flora circulating through. The ambient environment for PHA degradation in soil

422 was loam category having more moisture, humus, nutrients, and diverse soil biota, indicating

423 increased fertility. The effect of ambient environment on PHA, PLA and PHA-PLA polymers

424 investigated by soil-burial tests showed that degradation of PLA films was highest and remained

425 almost constant after weight reduction from $0.1 \mathrm{~g}$ to $0.02 \mathrm{~g}$ at 20 weeks. PHA-PLA

426 blendsfollowed these degradative rates closely. While comparative degradative ability of PHA

427 was lowest among all samples. Corn based PLA and PHA serves as carbon source for soil

428 microbiota. Among these PLA is most easily available and accessible to microorganisms as it

429 can be degraded both at the surface and under the surface enzymatically. High degradation of

430 PLA may also be due to its amorphous nature. Modal et al. reported $88 \%$ weight loss in

431 starch/sucrose composite films within 30 days of soil burial (Mondal 2015). Marichelvam et al.

432 reported $48.73 \%$ biodegradability in corn derived bioplastics after placement in soil at $3 \mathrm{~cm}$ 
433 depth for 15 days (Marichelvam et al 2019). PHA, on the other hand, was degraded on the 434 surface. It was speculated that rapid degradation of PHA films, while being high, was 435 comparatively lower since depolymerase enzymes only acted on the surface. Ong et al. reported 436 almost complete degradation of $0.03 \mathrm{~mm}$ PHA films after 8 weeks in soil (Ong and Sudesh 437 2016). However, among PHA samples degradation of mixed sample PHA (PM) was highest 438 indicating that it is also possible degradation of pure PHB homopolymer having higher 439 crystallinity was lower as compared degradation of comapartively less crystalline PHB 440 heteropolymer. Ong et al.also reported faster degradation of $\mathrm{P}(3 \mathrm{HB}-\mathrm{co}-21 \mathrm{~mol} \% 3 \mathrm{HHx})$ as 441 compared to $\mathrm{P}(3 \mathrm{HB})(\mathrm{Ong}$ and Sudesh 2016).Degradative ability of PHA-PLA blends can 442 therefore be high compared to PHA, as PLA phase is degraded, unveiling underlaying PHA 443 phase for degradation both on and under the surface. It is also possible that diverse microbiota of 444 soil environment degraded PHA by action of depolymerases extracellularly as well as 445 intracellularly by absorbing PHA granules. In degradative studies, it has been reported that 446 intracellular n-PHB depolymerase of Bacillus megaterium also exhibits extracellular activity 447 (Ray and Kalia 2017a).Previous studies have also reported that for polymer blend degradation 448 additive rule cannot be applied often. Thus making it difficult to predict polymer blend 449 degradation pattern based on the degradative behavior of pure components (La Mantia et al 450 2017).

451 SEM micrographs of PHA, PLA and PHA-PLA copolymer after degradation in soil showed the 452 presence ofdistinctive depolymerized fracture surfaces. Bacterial colonization of PHA, PHA453 PLA samples and additional fungal penetration of PLA samples leading to bursting and swelling, 454 probably aided in release of smaller molecules and/or short molecular chains capable of passing 455 through outer cell membranes. Ong et al. reported similar findings while studying effects of PHA 
456 degradation. They also attributed weight loss to bacterial and fungal colonization (Ong and 457 Sudesh 2016).PLA sample was shown to be degraded as a whole, indicating a nonenzymatic 458 degradation by hydrolysis. While presence of bacterial colonies and fungal hyphae on films 459 indicated an enzymatic degradation and uptake of degraded products. PHA sample, on the other 460 hand, showed occurrence high degradation at sample surfaces indicating an enzymatic 461 degradation following bacterial colonization. Ong et al. also reported catalytic action of 462 depolymerase enzymes on porous sample surfaces already degraded by surface erosion (Ong and 463 Sudesh 2016). PHA-PLA blends showed increased degradation compared to PHA, but decreased 464 degradation compared to PLA, accounting for the fact that both enzymatic and nonenzymatic 465 degradation occurred in the copolymer blends. SEM micrographs of PHA-PLA blends show that 466 erosion across the surface is due to PHA particles and degradation within the film structure is 467 due to PLA particles. Decreased degradation rate compared to PLA can be due to the uniform

468 distribution of PHA crystals across the PLA matrix. These PHA crystals can be degraded by 469 various surface enzymes but only after the overlaying PLA matrix has been degraded 470 nonenzymatically.

\section{Conclusion}

472 PHA-PLA blends show significant degradation.Physical blending of biologically produced PHA 473 using previously isolated PHA producers with chemically produced corn based PLA 474 significantly enhanced degradative ability of resulting blends. Biopolymers PHA, PLA and 475 PHA-PLA blends have significance in environmental studies as well as industrial sectors. 476 Knowing the impact of natural soil environment on biodegradable biopolymers will aid in 477 understanding the role of ambient microbial population in degradation. Degradation in different 
478 systems also opens new avenue of research to explore metabolic pathways of ambient polymer

479 degrading bacteria. Future studies can be focused on the effect of such degradative behaviors on 480 environment i.e. on soil fertility, irrigation water, etc.

\section{Materials and Methods}

\section{$482 \quad 5.1 \quad$ Revival of Stock Cultures}

483 Stock cultures of bacterial strains PWA; Bacillus subtilis (MH142143), PWC; Pseudomonas 484 aeruginosa (MH142144), PWF; Bacillus tequilensis (MH142145), PWG; Bacillus safensis 485 (MH142146), and DL3; Bacillus subtilis (MT043898) were taken from Research Lab II, MMG 486 (Sohail et al 2020; Sohail and Jamil 2019) and revived by culturing at $37^{\circ} \mathrm{C}$ on Nutrient Agar for 487 24h. Fresh cultures were maintained by streaking discrete colonies on Nutrient Agar and 488 incubating for 24 hours at $37^{\circ} \mathrm{C}$.

$489 \quad 5.2$ Polyhydroxyalkanoate Production

490 Polyhydroxyalkanoate (PHA) detection media supplemented with $2 \%$ glucose as carbon source 491 was used for cultivation of PHA producers (Chaudhry et al 2011; Javaid et al 2019; Rehman et al 492 2007). Preliminary $10 \%$ bacterial culture was added aseptically to $200 \mathrm{ml}$ media dispensed in $493500 \mathrm{ml}$ Erlenmeyer flasks and incubated at $37^{\circ} \mathrm{C}$ for 96 hours in a rotary incubator. Using 494 IRMECO U2020 UV-Vis spectrophotometer, optical densities were recorded at $600 \mathrm{~nm}$ (Teeka 495 et al 2010). Culture media ( $40 \mathrm{ml})$ was collected at 24, 48, 72 and 96 hours, and centrifuged at $4966000 \mathrm{rpm}$ for 15 minutes to obtain cell biomass. Cell biomass was measured in grams. PHA 497 produced by bacterial cells was extracted from biomass by SDS/ Sodium hypochlorite digestion 
498 and subsequent incubation in chloroform (Van Doan et al 2015). Pellet was treated at $25^{\circ} \mathrm{C}$ and

$499 \mathrm{pH} 10.0$ for 15 minutes with $0.25 \%$ SDS then for 5 minutes with $5.25 \%$ sodium hypochlorite and

500 centrifuged at $6000 \mathrm{rpm}$ for 15 minutes. Pellet was washed using ice cold acetone, centrifuged

501 again at $6000 \mathrm{rpm}$ for 15 minutes and transferred to $100 \mathrm{ml}$ glass Erlenmeyer flask. Chloroform

502 (10 volumes) was dispensed in flasks and incubated at room temperature for 48 hours.

503 Chloroform/PHA mixture was filtered using Whatman Grade 1 filter paper to remove cell debris.

504 Chloroform was evaporated to obtain solvent-cast dry films of PHA under a fume hood (Sohail

505 et al 2020; Sohail and Jamil 2019). Dry weight PHA films was recorded in grams (Van Doan et

506 al 2015). Percentage of PHA was calculated using formula:

507

$$
\% \text { PHA }=\frac{\text { WeightofPHA }}{\text { Weightofbiomass }} \times 100
$$

\section{$508 \quad 5.3$ Corn based Polylactide Production}

509 Corn starch was used for chemical production of corn-based polylactide (Keziah et al 2018;

510 Marichelvam et al 2019; Muller et al 2017). Corn starch (9.5 ml) was charged in $60.0 \mathrm{ml}$ of cold

511 deionized water (DI), $0.1 \mathrm{M} \mathrm{HCl}(2.0 \mathrm{ml})$, glycerin $(5.0 \mathrm{ml})$ used as plasticizer and $5 \%$ acetic

512 acid $(5.0 \mathrm{ml})$ and stirred vigorously until a uniform solution was prepared. Hot plate with

513 magnetic rotor was preheated to $120^{\circ} \mathrm{C}$ temperature for 10 minutes. Solution was placed on hot

514 plate under fume hood to avoid fumes, stirred vigorously and temperature was increased

515 gradually to $145^{\circ} \mathrm{C}$ over $20-25 \mathrm{~min}$. Mixture was stirred manually and agitated continuously till

516 a clear, homogeneous, gelatin-like suspension was formed(Basnet and Wiberg 2016). This semi

517 solid suspension was poured on aluminum foil sheet and spread uniformly using glass rod to

518 make a film. Cast film was air dried for 3-4 days to remove moisture (EstructuraProteinas 2020;

519 Green Plastics 2012; Rudnik 2019; Solomonides 2016). 


\subsection{Production of PHA-PLA Blends}

521 PHA polymer $(0.05 \mathrm{~g})$ was dissolved in chloroform $(5.0 \mathrm{ml})$ and air dried until $0.1 \mathrm{ml}$ 522 chloroform remained. PLA polymer (0.05) was dissolved in hot distilled water $(5.0 \mathrm{ml})$ at $65^{\circ} \mathrm{C}$

523 and volume was raised up to $19.9 \mathrm{ml}$. Distilled water containing PLA (19.9 ml) was dispensed in

524 chloroform containing PHA $(0.1 \mathrm{ml})$ at $25^{\circ}$ Cand mixed thoroughly to obtain 1:1 PHA-PLA

525 blend (Kirchnerová and Cave 1976; Karaffa 2013; Mackay et al 1980). Solution was dispensed 526 in glass vial, dried by evaporation with constant shaking at $25^{\circ} \mathrm{C}$. Final PHA-PLA blend of $0.1 \mathrm{~g}$ 527 weight was obtained as film.

\section{$528 \quad 5.5$ Characterization of PHA, PLA and PHA-PLA Blends}

529 Analyzation of structure, fracture surfaces, crystallinity, and identification of organic functional

530 groups of PHA, PLA and PHA-PLA blends were done using light microscopy, scanning electron

531 microscopy, FTIR spectroscopy, and tensile strength measurement. Light microscope,

532 LABOMED, was used to analyze structure of PLA, PHA and PHA-PLA blends. Light

533 micrographs were taken using IRMECO camera. For further clarification of sample morphology,

534 samples were stained using $10 \%$ dilution of crystal violet and $10 \%$ dilution of safranin for 1

535 minute. Samples were flooded with dye for 1 minute, washed using distilled water and air dried.

536 Unstained, crystal violet stained, and safranin stained samples were observed using 4 X, 10 X, 40

$537 \mathrm{X}$ and $100 \mathrm{X}$ magnification. Tensile strength of polymers was measured by calculating stress.

538 Scanning electron microscope, EVO LS 10, used to analyze morphologic characteristics i.e.

539 structure, crystallinity, fracture surfaces etc. of PLA, PHA and PHA-PLA blends, was operated

540 at $20.0 \mathrm{kV}$ acceleration voltage. Scanning electron microscopy was conducted at Central

541 Research Lab, Lahore Women's University. Samples were observed at 500 X, 1000 X, 2000 X 
542 and $3000 \mathrm{X}$ magnification under low vacuum (80 Pa) using Zeiss SE microscope.FT-IR analysis

543 was used to inspect presence of functional organic groups in PHA, PLA and PHA-PLA samples.

544 FT-IR absorption spectra were obtained at room temperature using SHIMADZU IRTracer-100.

545 FT-IR scan range was $4000 \mathrm{~cm}^{-1}$ to $400 \mathrm{~cm}^{-1}$. Resolution of $4 \mathrm{~cm}^{-1}$ was used to record all spectra

546 for an average of 64 scans. Data analysis was done using GRAM/AI software and percentage

547 crystallinity of polymer material was evaluated using the method suggested byZerbi(Zerbi et al 548 1989).

$$
\% \text { Crystallinity }=100-\left[1-\frac{\frac{I a}{1.2333 I b}}{1+\left(\frac{I a}{I b}\right)}\right] \times 100
$$

550 where $I a$ and $I b$ are the absorbance values determined from the bands at (PLA) $1745 \mathrm{~cm}^{-1}$ and $5511845 \mathrm{~cm}^{-1}$, (PF, M) $1732 \mathrm{~cm}^{-1}$ and $1832 \mathrm{~cm}^{-1}$, (PM) $1730 \mathrm{~cm}^{-1}$ and $1830 \mathrm{~cm}^{-1}$ or (F) $1728 \mathrm{~cm}^{-1}$ and $5521828 \mathrm{~cm}^{-1}$ respectively(Javaid et al 2019).

\subsection{Degradative Analysis of PHA, PLA and PHA-PLA Blends}

554 Bacterial degradation and environmental degradation analysis were conducted using thirteen 555 samples of PHA (6), PLA (1) and PHA-PLA (6) blends with even surface and $0.1 \mathrm{~g}$ weight. Six

556 PHA samples included PHA produced by strain PWA, PWC, PWF, PWG, DL3 and mixed 557 culture on glucose, labelled as A, C, F, G, DL3 and M. Six PHA-PLA blends were labelled as 558 PA, PC, PF, PG, PDL3 and PM for PHA sample of PWA, PWC, PWF, PWG, DL3 and mixed 559 culture, respectively. All PHA and PHA-PLA films were prepared by solvent-cast technique. 560 Polymer solution was poured in aluminum foil tray with the dimensions $1.0 \mathrm{~cm}$ x $1.0 \mathrm{~cm}$. Films 561 had $1.5 \mathrm{~mm}$ thickness and $0.1 \mathrm{~g}$ weight. In case of PLA, cast film was cut into small pieces 562 having the dimensions $1.0 \mathrm{~cm} \times 1.0 \mathrm{~cm}$. PLA films had a thickness of $1.0 \mathrm{~mm}$ and weight of 0.1 
563 grams. Only samples with even surface area were selected for studies. For each experiment to

564 study degradation, all thirteen samples were prepared in triplicate. Before being used for 565 experiments, samples were aged for 2 weeks.

\section{5.6.1 Degradation in DifferentEnvironmental Systems}

567 Degradation of PHA, PLA and PHA-PLA blends in different environmental systems i.e. soil, air, 568 water, and heat were performed during start of September 2019 to end of January 2020 at the

569 Department of Microbiology and Molecular Genetics, University of the Punjab.. In case of soil 570 systems, samples were placed in wire mesh carefully and buried in a 20 inches deep hole dug at 571 the base of Syzygium cumini (Jamun) tree. Study region was Study location was flat ground with 572 brown coloration and smooth clay loam type soil. The average $\mathrm{pH}$ of soil was 4.5 and average 573 temperature range during study was between $26^{\circ} \mathrm{C}$ to $28^{\circ} \mathrm{C}$. Soil microbial counts were $3.55 \mathrm{x}$ $57410^{6}, 3.58 \times 10^{6}, 3.62 \times 10^{6}, 3.65 \times 10^{6}$, and $3.70 \times 10^{6} \mathrm{CFU} / \mathrm{ml}$ for $10^{0}$ dilution during September 575 to January. In case of air, samples were placed openly in test tubes at sites with maximum air 576 distribution at MMG Research Lab II. Microbial counts in air were $2.52 \times 10^{2}, 2.36 \times 10^{2}, 2.47$ x $57710^{2}, 2.22 \times 10^{2}$, and $2.34 \times 10^{2} \mathrm{CFU} / \mathrm{m}^{3}$ during September to January. Samples were placed at 578 room temperature. In case of water, samples were placed in test tubes containing $10.0 \mathrm{ml}$ 579 untreated tap water having $\mathrm{pH} 8.2$ and average temperature $25^{\circ} \mathrm{C}$ and a microbial count of 208 $580 \mathrm{CFU} / \mathrm{ml}$. under semi-aerobic conditions. In case of heat, samples in test tubes were subjected to 581 anaerobic degradation at constant temperature of $37^{\circ} \mathrm{C}$ in an incubator. 


\subsubsection{Bacterial Degradation in Laboratory}

583 Bacterial degradation was analyzed through a modified methodology combining two methods

584 i.e. clear zone test (Nishida and Tokiwa 1993; Augusta et al 1993) and enzymatic degradation

585 via mineralization (Tokiwa and Calabia 2004; Shah et al 2008; Ray and Kalia 2017b).

586 Degradation of PHA, PLA and PHA-PLA blends by Bacillus subtilis and Pseudomonas

587 aeruginosa were observed. $\mathrm{N}$ agar media $(150.0 \mathrm{ml})$ was poured in $150 \mathrm{~mm}$ x $20 \mathrm{~mm}$ Pyrex®

588 glass petri dishes. $\mathrm{N}$ agar plates were used to prepare lawn of bacterial culture. Samples were 589 placed on $\mathrm{N}$ agar plates. Plates were incubated at $37^{\circ} \mathrm{C}$ for 12 weeks.

\section{$590 \quad$ 5.6.3 Analysis of Samples During and After Degradation}

591 For bacterial degradation, changes in sample morphology, sample color, changes in bacterial

592 growth surrounding sample and changes in media surrounding sample were noted over a period

593 of 12 weeks. For degradation in different environmental systems, changes in color and texture of

594 samples were noted at regular intervals over a period of 20 weeks, as an indicator of degradation

595 by physical appearance. Changes in fracture surfaces, crystallinity and overall microscopic

596 degradation of samples was observed using light microscopy and scanning electron microscopy.

597 Optical microscope, LABOMED, was used to observe sample degradation at $4 \mathrm{X}$ and 10X. Light

598 micrographs were taken using IRMECO camera Scanning electron microscopy was conducted at

$599500 \mathrm{X}, 1000 \mathrm{X}, 2000 \mathrm{X}$ and $3000 \mathrm{X}$ magnification under low vacuum (80 Pa) using Zeiss EVO

600 LS 10,operated at $20.0 \mathrm{kV}$ acceleration voltage.Degradation by weight over 20 weeks was used

601 as indication of sample biodegradation rate.For weight loss analysis, initial sample weight $\left(\mathrm{W}_{\mathrm{i}}\right)$

602 was set at 0.1 grams as starting point. Each sample formulation was prepared in triplicate for all

603 experiments. Samples were retrieved each week from sampling locations, rinsed with distilled 
604 water and dried overnight. Constant weight was achieved by placing dried samples in a 605 desiccator. Weight of each film was measured and recorded. Percentage weight change was 606 calculated as follow:

607

$$
\text { Percentage Weight Change }=\frac{\left(W_{m}-W_{i}\right)}{W_{i}} X 100
$$

608 whereas $W_{i}$ is the initial sample weight and $W_{m}$ is the measured sample weight after degradation 609 at regular intervals (Zhang and Thomas 2011; Ong and Sudesh 2016).

610 Differences in weight during degradation were analyzed by plotting weight (decigrams) against y

611 axis and time (weeks) against $\mathrm{x}$ axis. Mean values recorded during triplicate experiments were

612 used. Standard error and standard deviation for triplicate formulations of samples were 613 calculated.

\section{Declarations}

\section{6.1 Ethics approval and consent to participate}

616 Not applicable

\section{6.2 Consent for publication}

618 Not applicable

\section{6.3 Availability of data and materials}

620 All data generated or analysed during this study are included in this published article. 


\section{$621 \quad 6.4$ Competing interests}

622 The authors declare that they have no competing interest.

\section{$623 \quad 6.5 \quad$ Funding}

624 Not applicable

\section{$625 \quad 6.6$ Authors' contributions}

626 RS performed experiments and wrote manuscripts while NJ supervised, designed experiments

627 and edited manuscript. All authors read and approved the final manuscript.

\section{$628 \quad 6.7$ Acknowledgements}

629 Not applicable

\section{$630 \quad 6.8$ Authors' information}

631 Not applicable

\section{$\begin{array}{lll}632 & 7 & \text { References }\end{array}$}

633 Aljuraifani AA, Berekaa MM, Ghazwani AA (2019) Bacterial biopolymer

634 (polyhydroxyalkanoate) production from low-cost sustainable sources. MicrobiologyOpen

635 8(6): 00755

636 Augusta J, Müller R-J, Widdecke H (1993) A rapid evaluation plate-test for the biodegradability

637 of plastics. Appl Microbiol Biotechnol 39(4-5):673-678

638 Ausejo JG, Rydz J, Musioł M et al (2018) A comparative study of three-dimensional printing 639 directions: The degradation and toxicological profile of a PLA/PHA blend. Polym Degradation $640 \quad$ Stab 152:191-207 
641 Basnet S, Wiberg B (2016) Production of Polylactic acid in laboratory scale, and characterising

642 the thermal properties. Degree thesis, Arcada University of Technology.

643 Chaudhry WN, Jamil N, Ali I et al (2011) Screening for polyhydroxyalkanoate (PHA)-producing

644 bacterial strains and comparison of PHA production from various inexpensive carbon sources.

645 Ann Microbiol 61(3):623-629

646 EstructuraProteinas (2020) Bioplastic from starch - home made.

647 https://www.youtube.com/watch?v=y1joh_t1thc\&t=19s. Accessed 29 Jan 2020

648 Fossi MC, Vlachogianni T, Galgani F et al (2020) Assessing and mitigating the harmful effects

649 of plastic pollution: the collective multi-stakeholder driven Euro-Mediterranean response. Ocean

650 Coast Manage 184(2020):105005

651 Gopi S, Kontopoulou M, Ramsay BA et al (2018) Manipulating the structure of medium-chain-

652 length polyhydroxyalkanoate (MCL-PHA) to enhance thermal properties and crystallization

653 kinetics. Int J Biol Macromol 119(2018):1248-1255. doi:10.1016/j.ijbiomac.2018.08.016

654 Green Plastics (2012) The new science of bioplastic. Green-plastics.net website: https://green-

655 plastics.net/ Accessed 29 Jan 2020

656 Ismail NA, Mohd Tahir S, Yahya N et al (2016) Synthesis and characterization of biodegradable

657 starch-based bioplastics. In: Mater Sci Forum. Trans Tech Publ, p 673-678

658 Iwata T (2015) Biodegradable and bio-based polymers: future prospects of eco-friendly plastics.

659 Angew Chem Int Ed 54(11):3210-3215

660 Javaid N, Batool R, Jamil N (2019) Blend of Polyhydroxyalkanoates Synthesized By Lipase

661 Positive Bacteria From Plant Oils. Journal of Renewable Materials 7(5):460-473 
662 Johnston B, Jiang G, Hill D et al (2017) The molecular level characterization of biodegradable

663 polymers originated from polyethylene using non-oxygenated polyethylene wax as a carbon

664 source for polyhydroxyalkanoate production. Bioengineering 4(3):73

665 Karaffa LS (2013) The Merck index: an encyclopedia of chemicals, drugs, and biologicals. RSC 666 Publishing.

667 Keziah VS, Gayathri R, Priya VV (2018) Biodegradable plastic production from corn starch. 668 Drug Invent Today 10(7):1315-1317

669 Kirchnerová J, Cave GC (1976) The solubility of water in low-dielectric solvents. Can J Chem $670 \quad 54(24): 3909-3916$

671 Koller M (2018) Biodegradable and Biocompatible Polyhydroxy-alkanoates (PHA): Auspicious 672 Microbial Macromolecules for Pharmaceutical and Therapeutic Applications. Molecules 673 23(2):362. doi:10.3390/molecules23020362

674 Koller M, Dias MMdS, Rodríguez-Contreras A et al (2015) Liquefied wood as inexpensive 675 precursor-feedstock for bio-mediated incorporation of (R)-3-hydroxyvalerate into 676 polyhydroxyalkanoates. Materials 8(9):6543-6557

677 Kricheldorf HR (2001) Syntheses and application of polylactides. Chemosphere 43(1):49-54. 678 doi:10.1016/s0045-6535(00)00323-4

679 La Mantia F, Morreale M, Botta L et al (2017) Degradation of polymer blends: A brief review. 680 Polym Degradation Stab 145:79-92

681 Leja K, Lewandowicz G (2010) Polymer Biodegradation and Biodegradable Polymers-a Review. 682 Pol J Environ Stud 19(2):255-266

683 Li Z, Loh XJ (2015) Water soluble polyhydroxyalkanoates: future materials for therapeutic 684 applications. Chem Soc Rev 44(10):2865-2879. doi:10.1039/c5cs00089k 
685 Li Z, Yang J, Loh XJ (2016) Polyhydroxyalkanoates: opening doors for a sustainable future.

686 NPG Asia Mater 8(4):e265

687 Mackay D, Bobra A, Shiu W et al (1980) Relationships between aqueous solubility and octanol-

688 water partition coefficients. Chemosphere 9(11):701-711

689 Marichelvam M, Jawaid M, Asim M (2019) Corn and rice starch-based bio-plastics as alternative 690 packaging materials. Fibers 7(4):32

691 Masood F, Abdul-Salam M, Yasin T et al (2017) Effect of glucose and olive oil as potential

692 carbon sources on production of PHAs copolymer and tercopolymer by Bacillus cereus FA11. 3

693 Biotech 7(1):87

694 Modjarrad K, Ebnesajjad S (2013) Handbook of polymer applications in medicine and medical 695 devices. Elsevier.

696 Mondal R (2015) Synthesis and characterization of Starch based biocomposite films and their 697 degradation behavior--an alternative to the conventional polymer. Doctoral dissertation, National 698 institute of technology, Rourkela.

699 Msuya N, Katima J, Massanja E et al (2017) Poly (lactic-acid) Production-from Monomer to 700 Polymer: A review. J Polym Sci 1(1):

701 Muller J, González-Martínez C, Chiralt A (2017) Combination of poly (lactic) acid and starch for 702 biodegradable food packaging. Materials 10(8):952

703 Munir S, Jamil N (2018) Polyhydroxyalkanoates (PHA) production in bacterial co-culture using 704 glucose and volatile fatty acids as carbon source. J Basic Microbiol 58(3):247-254

705 Muniyasamy S, Muniyasamy S, Mohanrasu K et al (2019) Biobased Biodegradable Polymers for

706 Ecological Applications: A Move Towards Manufacturing Sustainable Biodegradable Plastic 707 Products. Integrating Green Chemistry \& Sustainable Engineering. 
708 Muthuraj R, Misra M, Mohanty AK (2018) Biodegradable compatibilized polymer blends for

709 packaging applications: A literature review. J Appl Polym Sci 135(24):45726

710 Narancic T, Verstichel S, Reddy Chaganti S et al (2018) Biodegradable plastic blends create new

711 possibilities for end-of-life management of plastics but they are not a panacea for plastic

712 pollution. Environ Sci Technol 52(18):10441-10452

713 Nerkar M, Ramsay JA, Ramsay BA et al (2015) Improvements in the melt and solid-state

714 properties of poly (lactic acid), poly-3-hydroxyoctanoate and their blends through reactive

715 modification. Polym Plast Technol Eng 64:51-61

716 Nishida H, Tokiwa Y (1993) Distribution of poly ( $\beta$-hydroxybutyrate) and poly ( $\varepsilon$-caprolactone)

717 aerobic degrading microorganisms in different environments. J Environ Polym Degrad 1(3):227-

$718 \quad 233$

719 Ong SY, Sudesh K (2016) Effects of polyhydroxyalkanoate degradation on soil microbial 720 community. Polym Degradation Stab 131:9-19

721 Pretula J, Slomkowski S, Penczek S (2016) Polylactides-Methods of synthesis and

722 characterization. Adv Drug Deliv Rev 107(2016):3-16

723 Radecka I, Irorere V, Jiang G et al (2016) Oxidized polyethylene wax as a potential carbon 724 source for PHA production. Materials 9(5):367

725 Rasal RM, Bohannon BG, Hirt DE (2008) Effect of the photoreaction solvent on surface and 726 bulk properties of poly(lactic acid) and poly(hydroxyalkanoate) films. J Biomed Mater Res B

727 Appl Biomater 85(2):564-572. doi:10.1002/jbm.b.30980

728 Ray S, Kalia VC (2017a) Biological significance of degradation of polyhydroxyalkanoates. In:

729 Microbial Applications, vol 1. Springer, Cham, p 125-139. doi:https://doi.org/10.1007/978-3-

730 319-52666-9_5 
731 Ray S, Kalia VC (2017b) Polyhydroxyalkanoate Production and Degradation Patterns in Bacillus

732 Species. Indian J Microbiol 57(4):387-392. doi:10.1007/s12088-017-0676-y

733 Reddy C, Ghai R, Kalia VC (2003) Polyhydroxyalkanoates: an overview. Bioresour Technol

$734 \quad 87(2): 137-146$

735 Rehman S, Jamil N, Husnain S (2007) Screening of different contaminated environments for

736 polyhydroxyalkanoates-producing bacterial strains. Biologia 62(6):650-656

737 Roy I, Visakh P (2014) Polyhydroxyalkanoate (PHA) based blends, composites and 738 nanocomposites, Vol 30. Royal Society of Chemistry.

739 Royte E (2006) Corn plastic to the rescue. Smithsonian Magazine 37(5):84-88.

740 https://www.smithsonianmag.com/science-nature/corn-plastic-to-the-rescue-126404720/

741 Rudnik E (2019) Compostable polymer materials. Second edn. Elsevier.

742 Sarasa J, Gracia JM, Javierre C (2009) Study of the biodisintegration of a bioplastic material

743 waste. Bioresour Technol 100(15):3764-3768. doi:10.1016/j.biortech.2008.11.049

744 Shah AA, Hasan F, Hameed A et al (2008) Biological degradation of plastics: a comprehensive

745 review. Biotechnol Adv 26(3):246-265. doi:10.1016/j.biotechadv.2007.12.005

746 Shah TV, Vasava DV (2019) A glimpse of biodegradable polymers and their biomedical

747 applications. epoly 19(1):385-410

748 Shogren R (2009) Starch-poly (hydroxyalkanoate) composites and blends. Biodegrad Polym

749 Blends Compos from Renew Resour, John Wiley \& Sons, Inc:211-226

750 Sintim HY, Bary AI, Hayes DG et al (2019) Release of micro- and nanoparticles from

751 biodegradable plastic during in situ composting. Sci Total Environ 675:686-693.

752 doi:10.1016/j.scitotenv.2019.04.179 
753 Sohail R, Jamil N (2019) Isolation of biosurfactant producing bacteria from Potwar oil fields:

754 Effect of non-fossil fuel based carbon sources. Green Process Synth 9(1):77-86

755 Sohail R, Jamil N, Ali I et al (2020) Animal fat and glycerol bioconversion to

756 polyhydroxyalkanoate by produced water bacteria. epoly 20(1):92-102

757 Solomonides EG (2016) Biodegradable bioplastic compositions and methods of making and

758 using the same.

759 Su S, Kopitzky R, Tolga S et al (2019) Polylactide (PLA) and Its Blends with Poly(butylene

760 succinate) (PBS): A Brief Review. Polymers (Basel) 11(7):1193. doi:10.3390/polym11071193

761 Tan G-YA, Chen C-L, Li L et al (2014) Start a research on biopolymer polyhydroxyalkanoate

762 (PHA): a review. Polymers 6(3):706-754

763 Teeka J, Imai T, Cheng X et al (2010) Screening of PHA-producing bacteria using biodiesel-

764 derived waste glycerol as a sole carbon source. J Water Environ Technol 8(4):373-381

765 Tokiwa Y, Calabia BP (2004) Review degradation of microbial polyesters. Biotechnol Lett 766 26(15):1181-1189

767 Van Doan T, Hoi LT, Phong TH (2015) Recovery of Poly (3-hydroxybutyrate) from Yangia sp.

768 ND199 by Simple Digestion with sodium hypochlorite. Vietnam Journal of Science and

769 Technology 53(6):706

770 Verma R, Vinoda K, Papireddy M et al (2016) Toxic pollutants from plastic waste-a review.

771 Procedia Environ Sci 35:701-708

772 Volova T, Boyandin A, Vasiliev A et al (2010) Biodegradation of polyhydroxyalkanoates

773 (PHAs) in tropical coastal waters and identification of PHA-degrading bacteria. Polym

774 Degradation Stab 95(12):2350-2359 
775 Yang S (2014) Novel bio-based and biodegradable polymer blends. Dissertation Thesis, Iowa

776 State University.

777 Zerbi G, Gallino G, Del Fanti N et al (1989) Structural depth profiling in polyethylene films by

778 multiple internal reflection infra-red spectroscopy. Polym Plast Technol Eng 30(12):2324-2327

779 Zhang J, Yan D-X, Xu J-Z et al (2012) Highly crystallized poly (lactic acid) under high pressure.

780 AIP Advances 2(4):042159

781 Zhang M, Thomas NL (2011) Blending polylactic acid with polyhydroxybutyrate: the effect on

782 thermal, mechanical, and biodegradation properties. Adv Polym Tech 30(2):67-79

783 Zinn M, Witholt B, Egli T (2001) Occurrence, synthesis and medical application of bacterial

784 polyhydroxyalkanoate. Adv Drug Deliv Rev 53(1):5-21. doi:10.1016/s0169-409x(01)00218-6 
$\begin{array}{lll}786 & 8 & \text { Figure Titles }\end{array}$

787 Figure 1 FT-IR Spectrum of PHA Sample of Strain PWF

788 Figure 2 FT-IR Spectrum of PHA Sample of Mixed Culture

789 Figure 3 FT-IR Spectrum of PLA Sample.

790 Figure 4 FT-IR Spectrum of PHA-PLA Sample of Strain PWF

791 Figure 5 FT-IR Spectrum of PHA-PLA Sample of Mixed Culture

792 Figure 6 SEM of PHA sample F (A), PLA (B) and PHA-PLA sample PF (C) before and after 793 degradation at $3000 \mathrm{X}$.

794 Figure 7 Graphical representation for soil degradation of PHA, PLA, and PHA-PLA blends.

795 Figure 8 Graphical representation for water degradation of PHA, PLA, and PHA-PLA blends.

796 Figure 9 Graphical representation for air degradation of PHA, PLA, and PHA-PLA blends.

797 Figure 10 Graphical representation for heat degradation of PHA, PLA, and PHA-PLA blends.

$\begin{array}{lll}798 & 9 & \text { Table Titles }\end{array}$

799 Table 1PHA Production on Glucose 
Figures

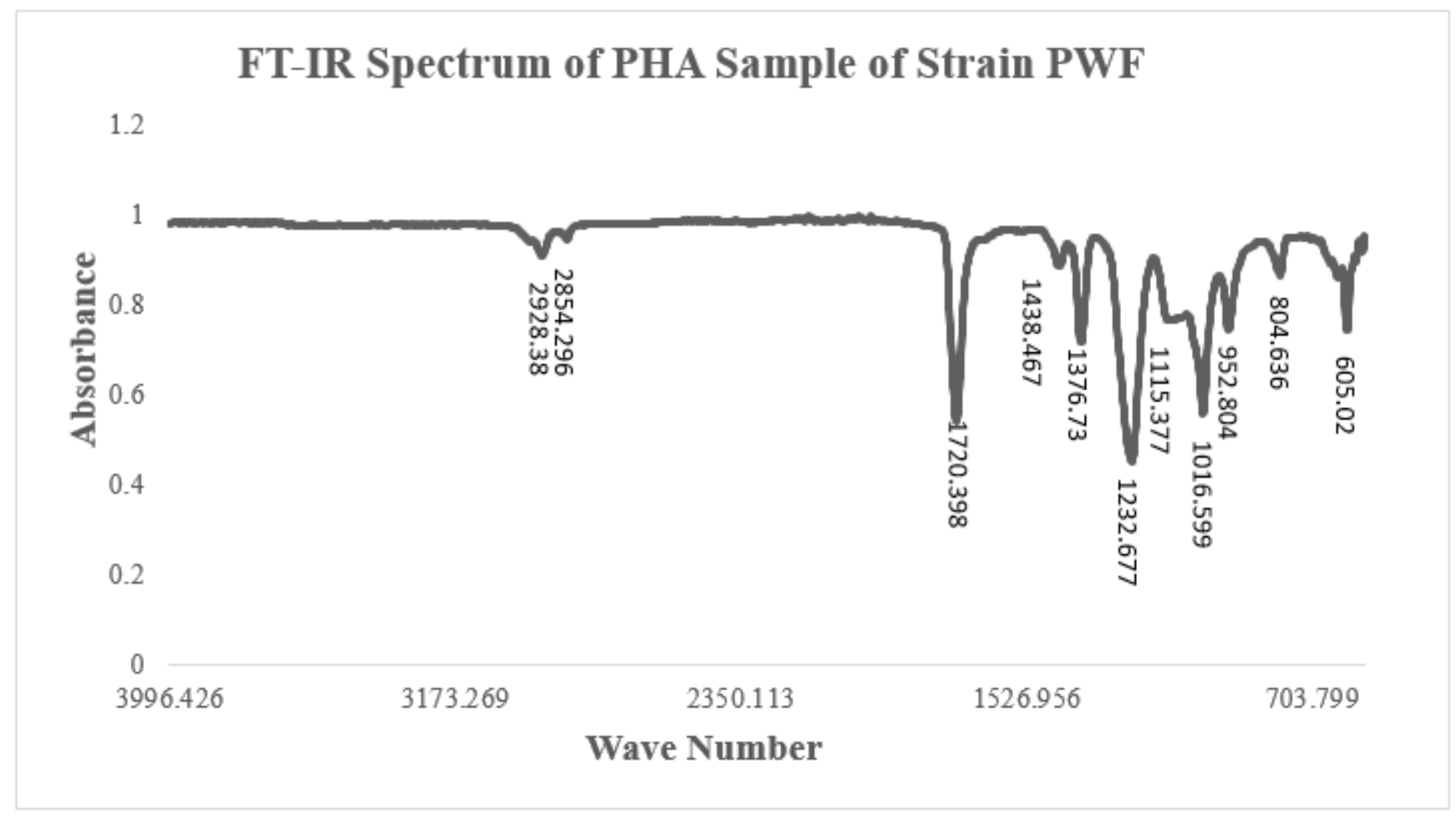

Figure 1

FT-IR Spectrum of PHA Sample of Strain PWF 
FT-IR Spectrum of PHA Sample of Strain PWF

1.2

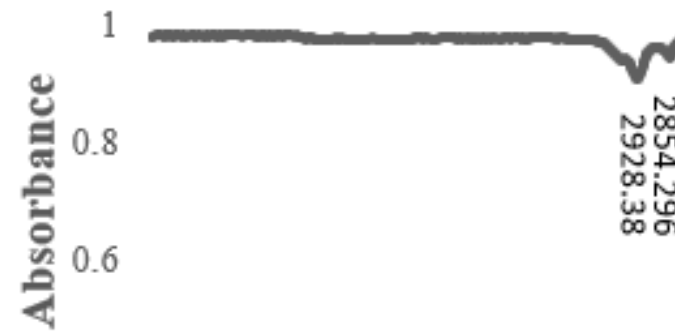

0.4

0.2

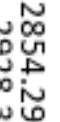

шొڤ̆

3996.426

3173.269

2350.113

1526.956

703.799

Wave Number

Figure 1

FT-IR Spectrum of PHA Sample of Strain PWF

FT-IR Spectrum of PHA Sample of Strain PWF

1.2

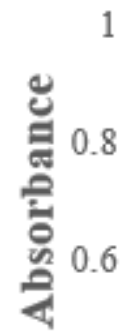

0.4

0.2

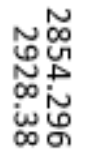

0

3996.426

3173.269

2350.113

1526.956

703.799

Wave Number 
Figure 1

FT-IR Spectrum of PHA Sample of Strain PWF

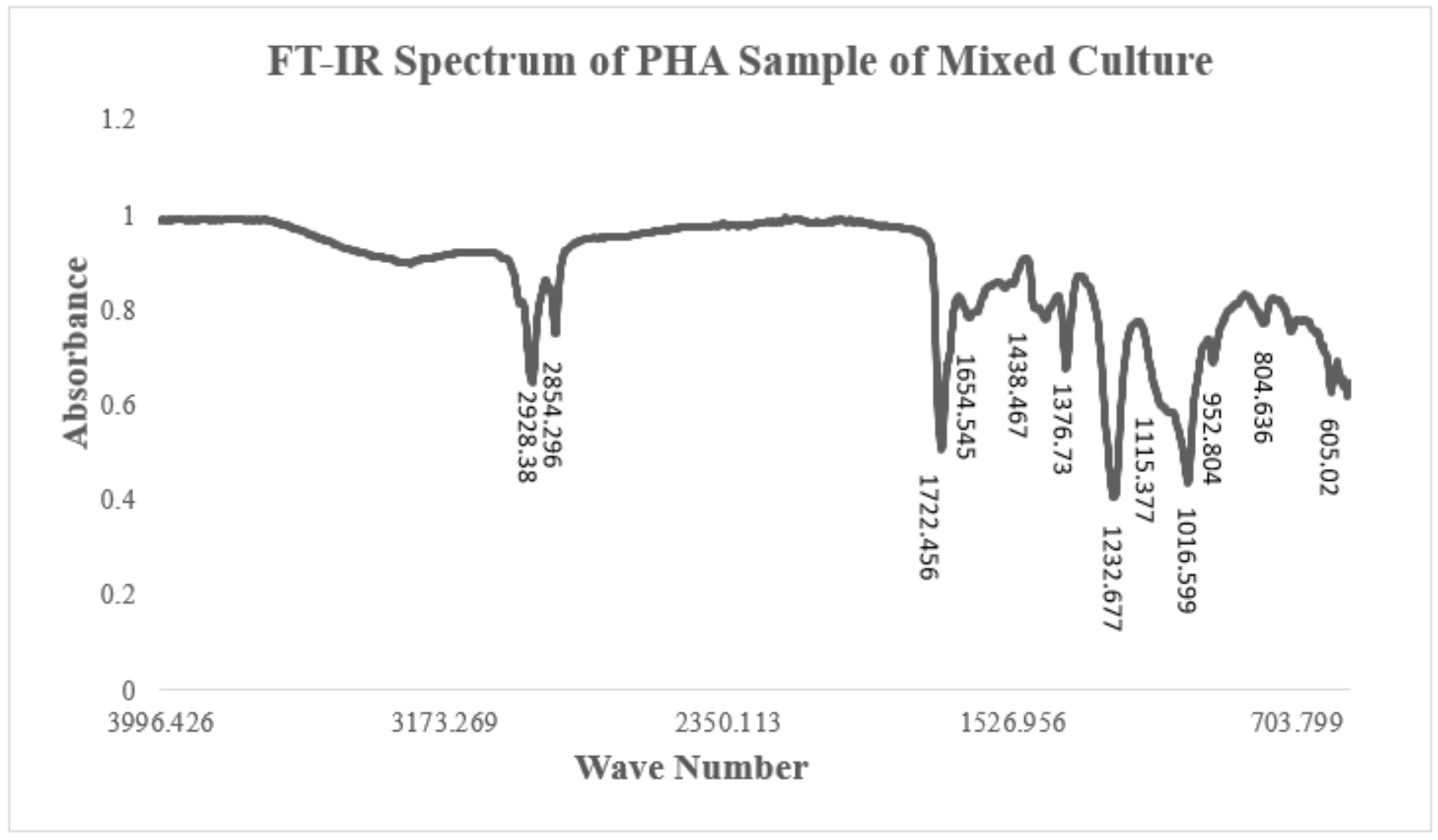

Figure 2

FT-IR Spectrum of PHA Sample of Mixed Culture 


\section{FT-IR Spectrum of PHA Sample of Mixed Culture}

1.2

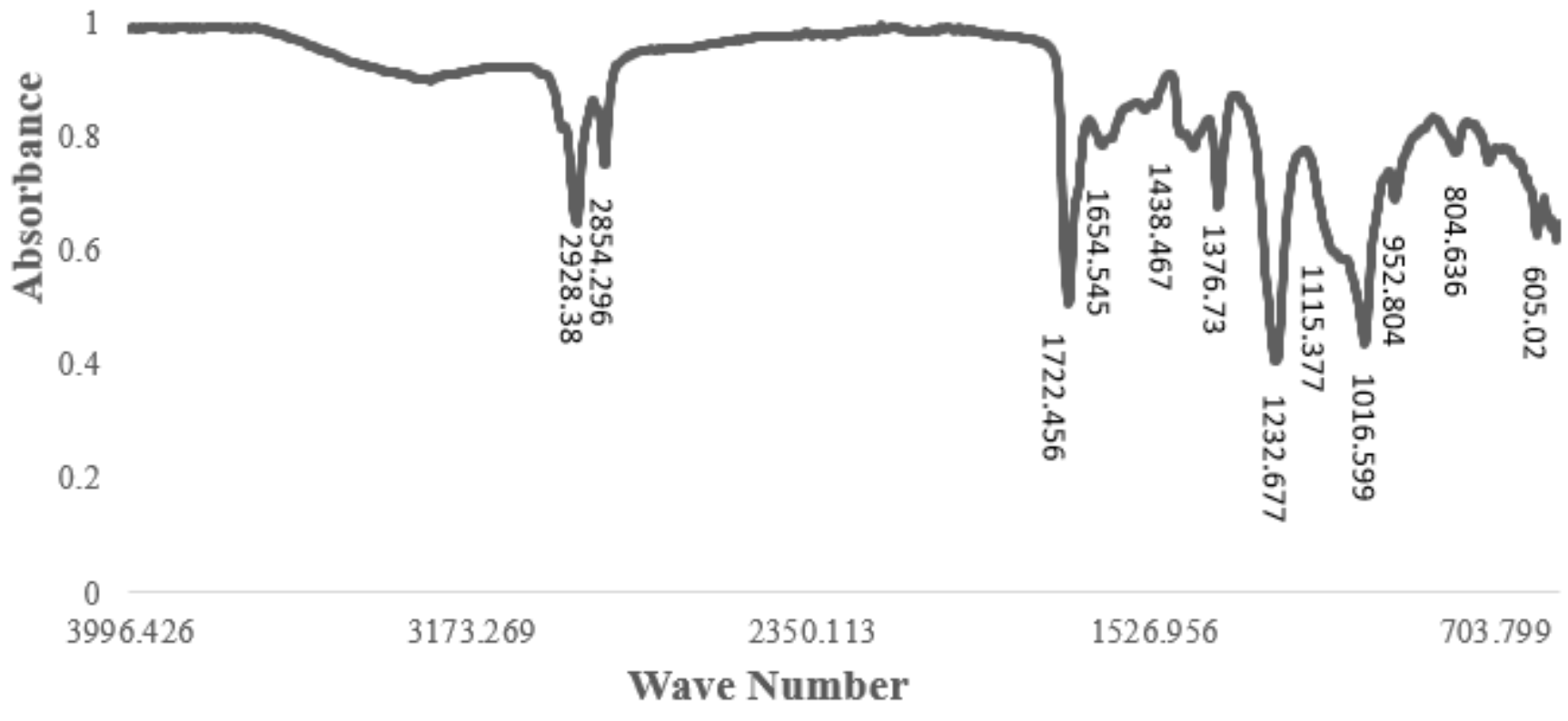

Figure 2

FT-IR Spectrum of PHA Sample of Mixed Culture

FT-IR Spectrum of PHA Sample of Mixed Culture

1.2

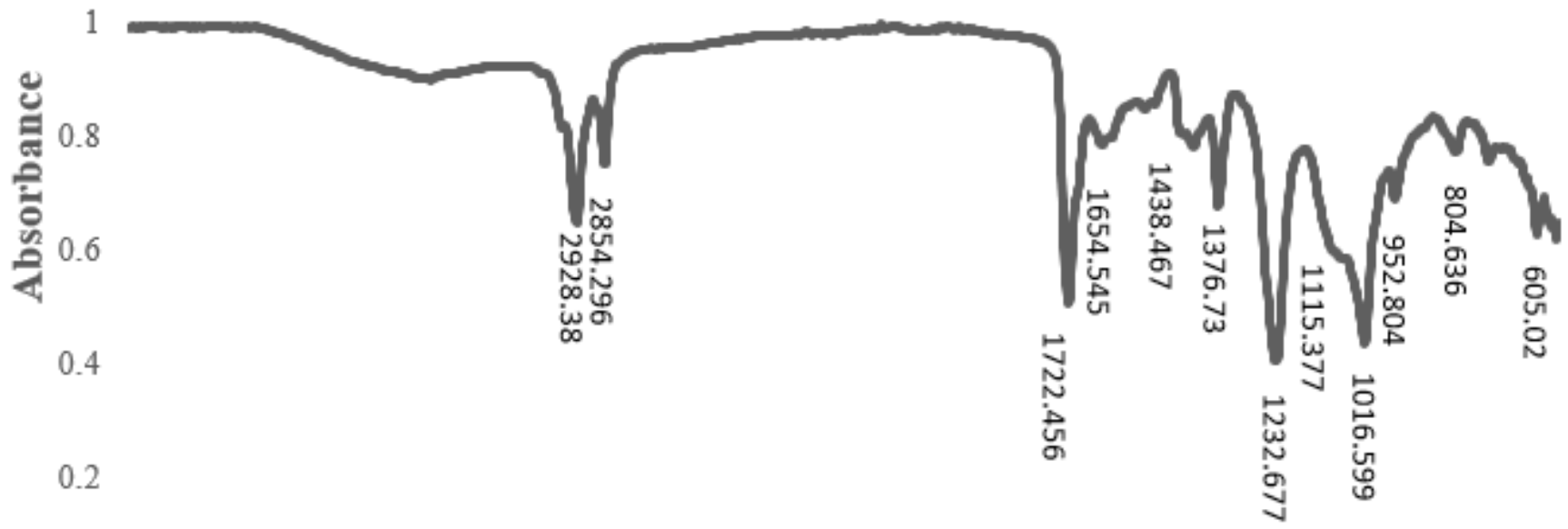

0 
Figure 2

FT-IR Spectrum of PHA Sample of Mixed Culture

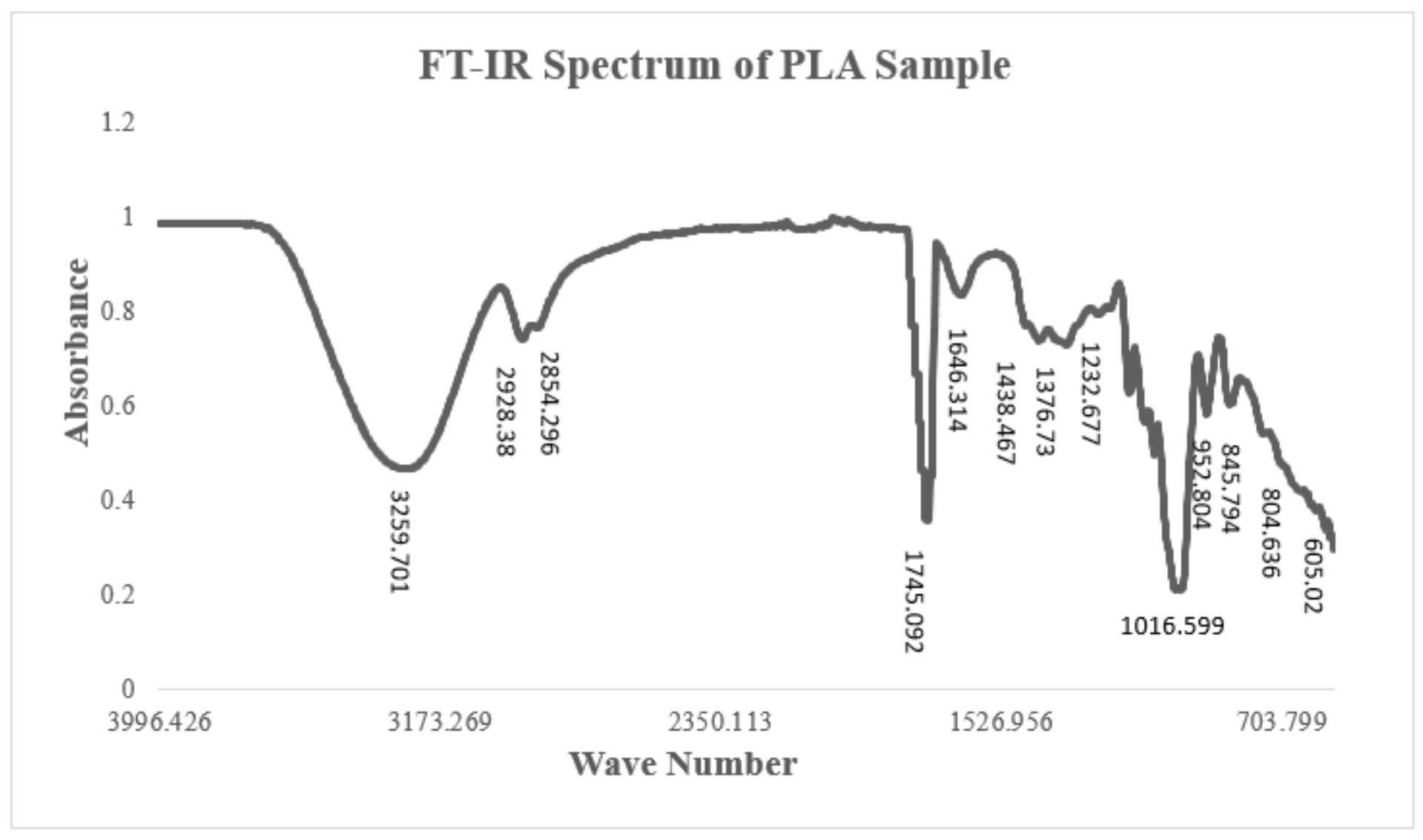

Figure 3

FT-IR Spectrum of PLA Sample. 


\section{FT-IR Spectrum of PLA Sample}

1.2

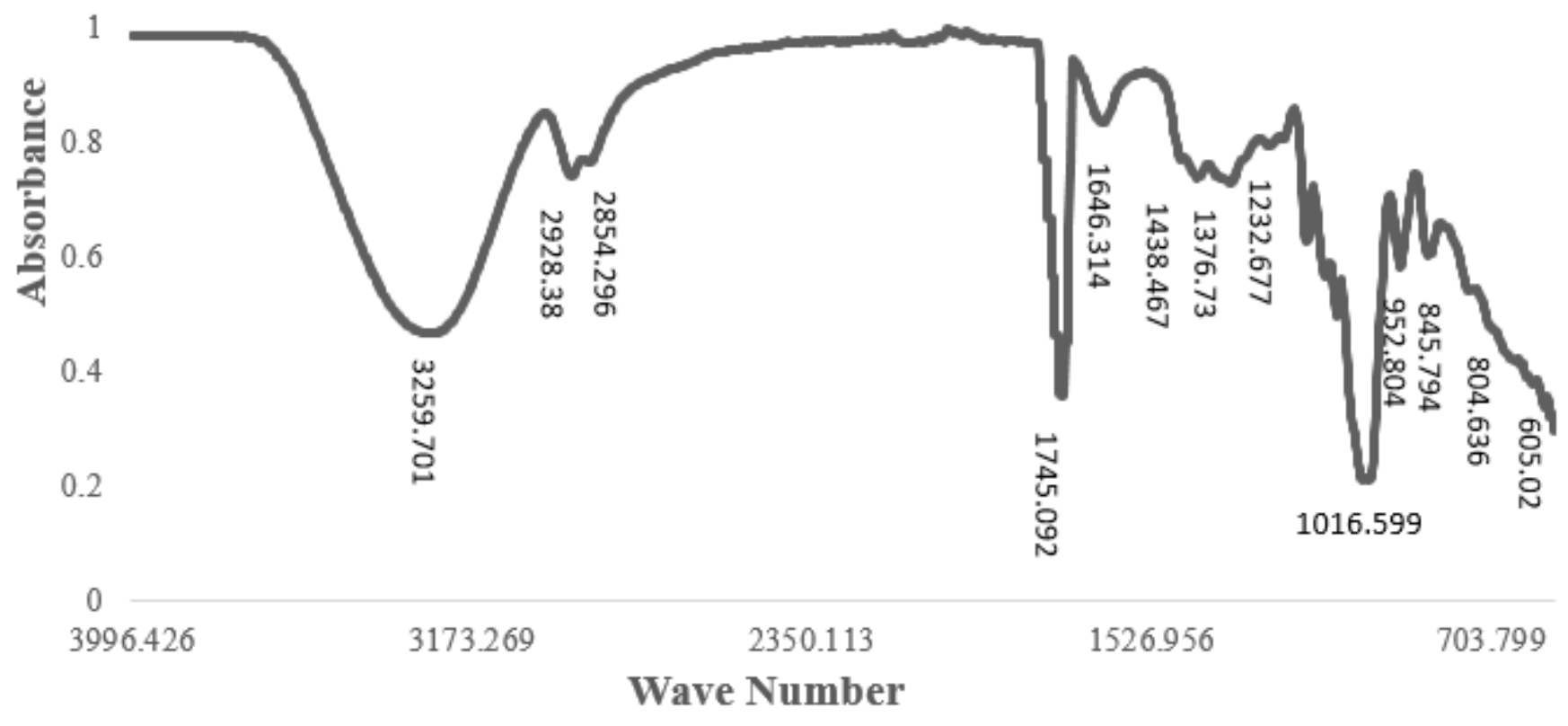

Figure 3

FT-IR Spectrum of PLA Sample. 


\section{FT-IR Spectrum of PLA Sample}

1.2

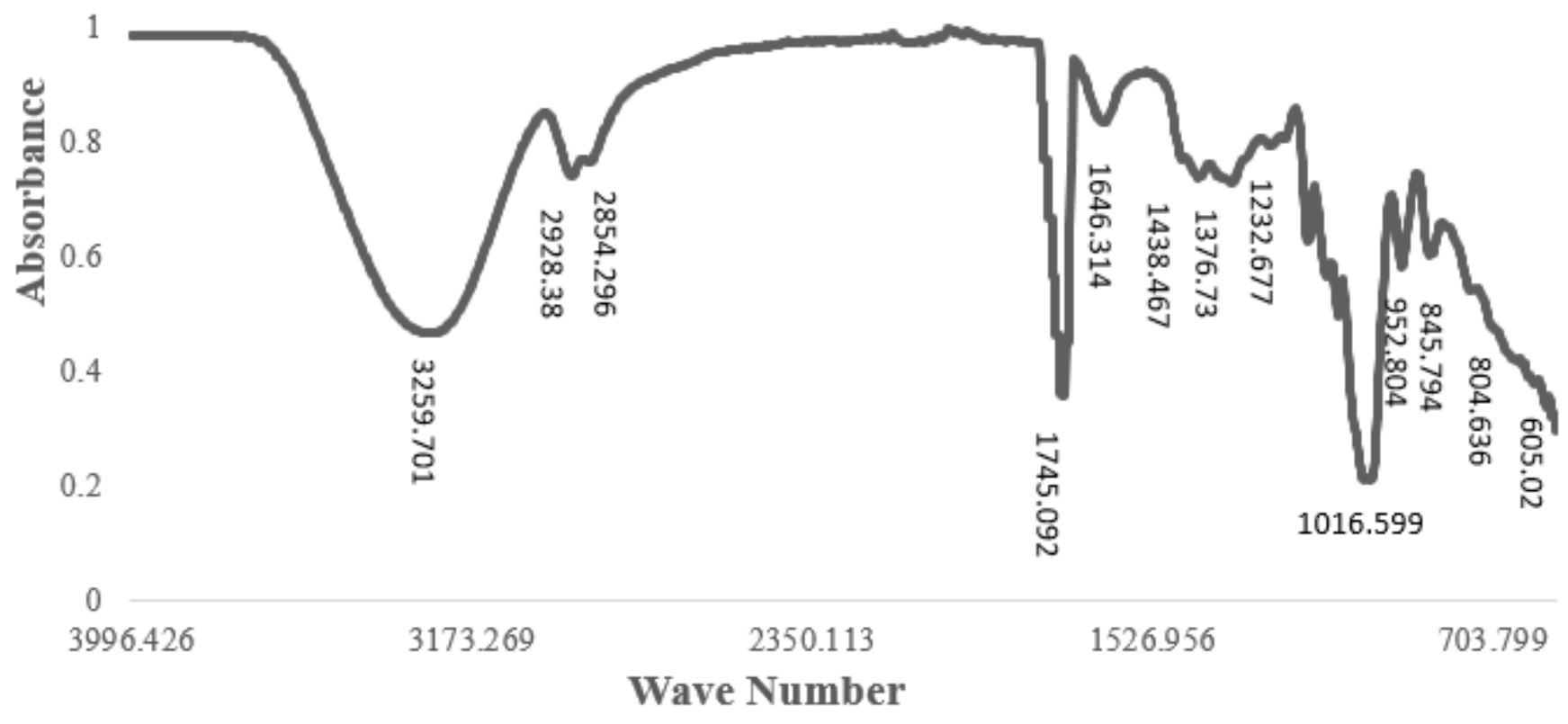

Figure 3

FT-IR Spectrum of PLA Sample.

FT-IR Spectrum of PHA-PLA Sample of Strain PWF

1.2

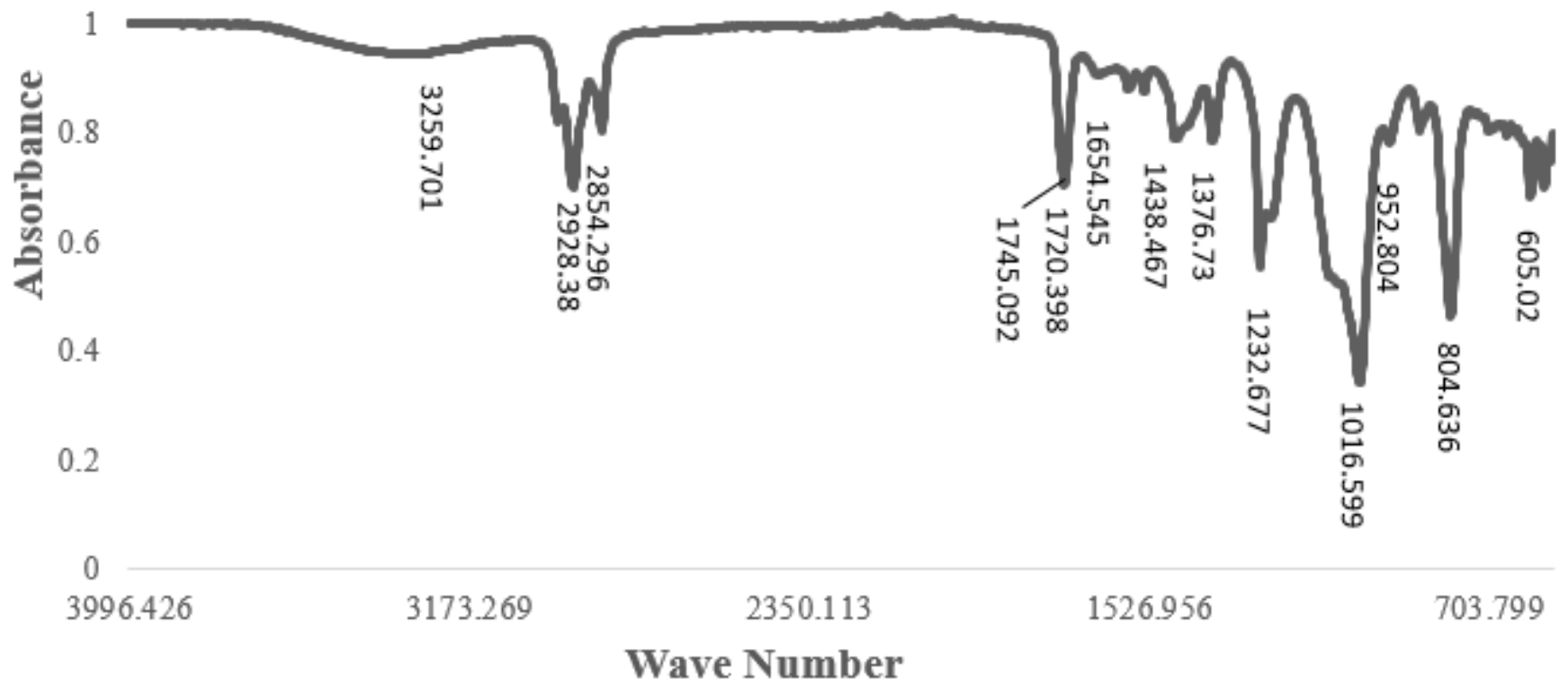


Figure 4

FT-IR Spectrum of PHA-PLA Sample of Strain PWF

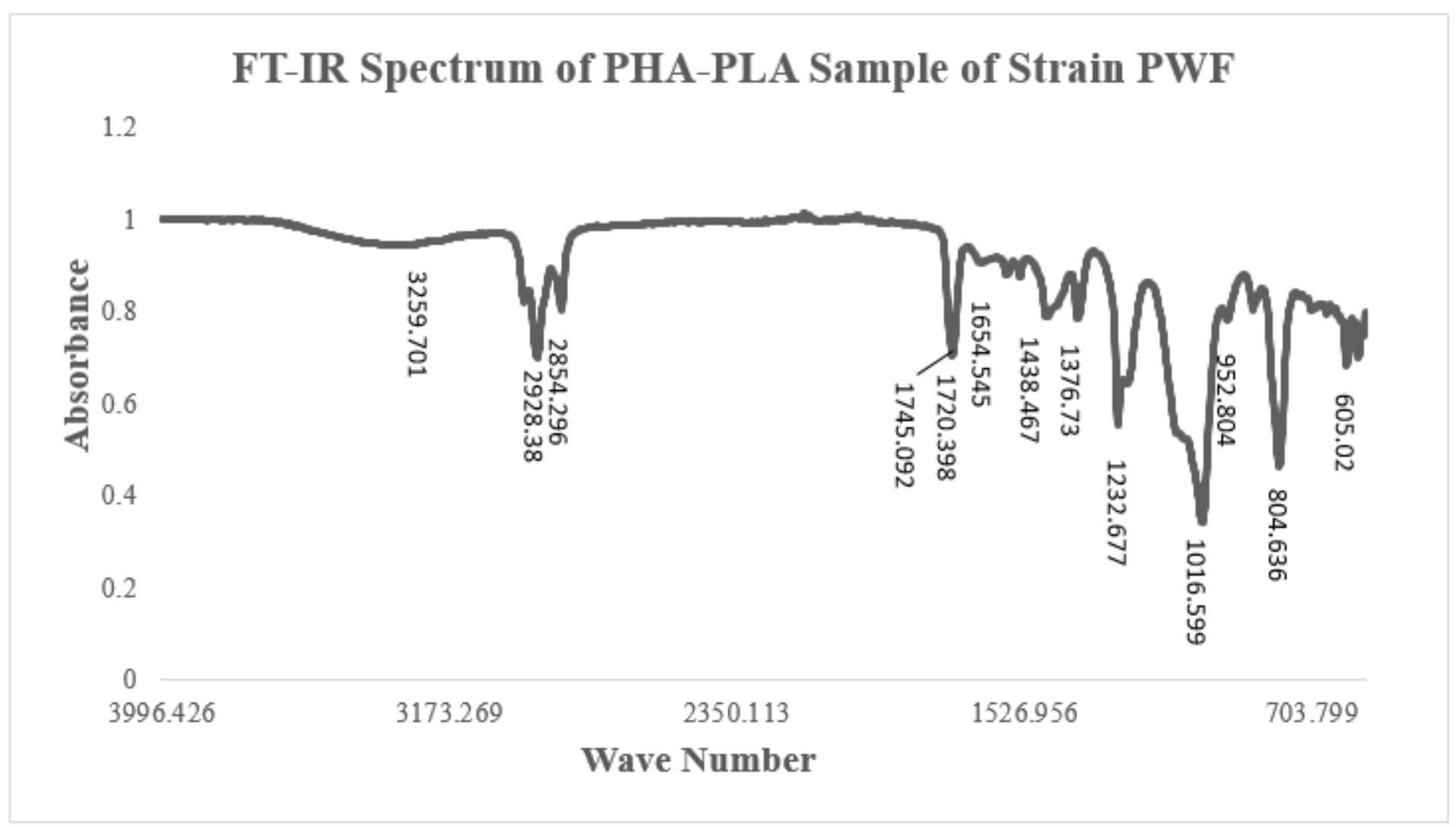

Figure 4

FT-IR Spectrum of PHA-PLA Sample of Strain PWF 


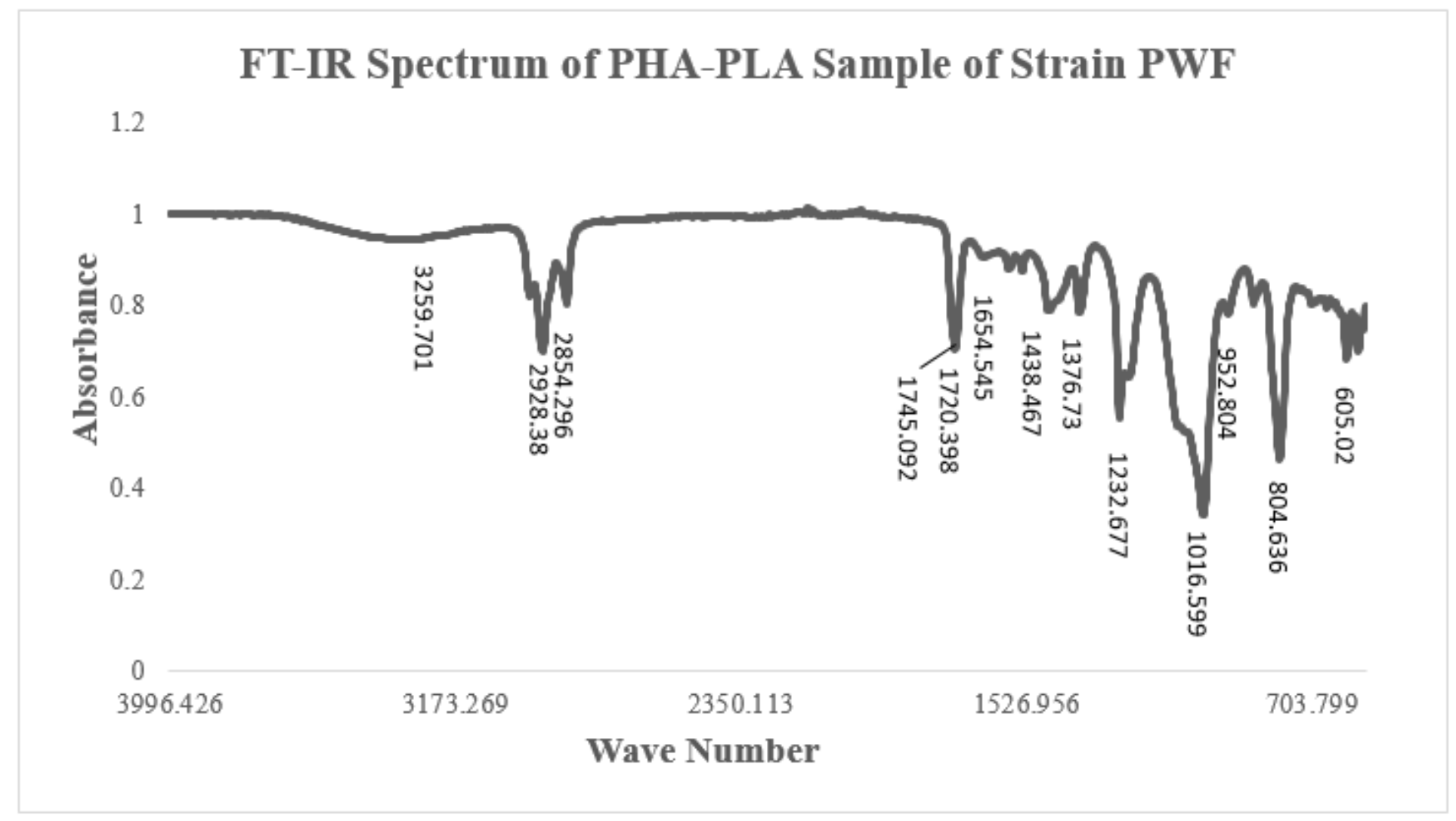

Figure 4

FT-IR Spectrum of PHA-PLA Sample of Strain PWF

FT-IR Spectrum of PHA-PLA Sample of Mixed Culture

1.2

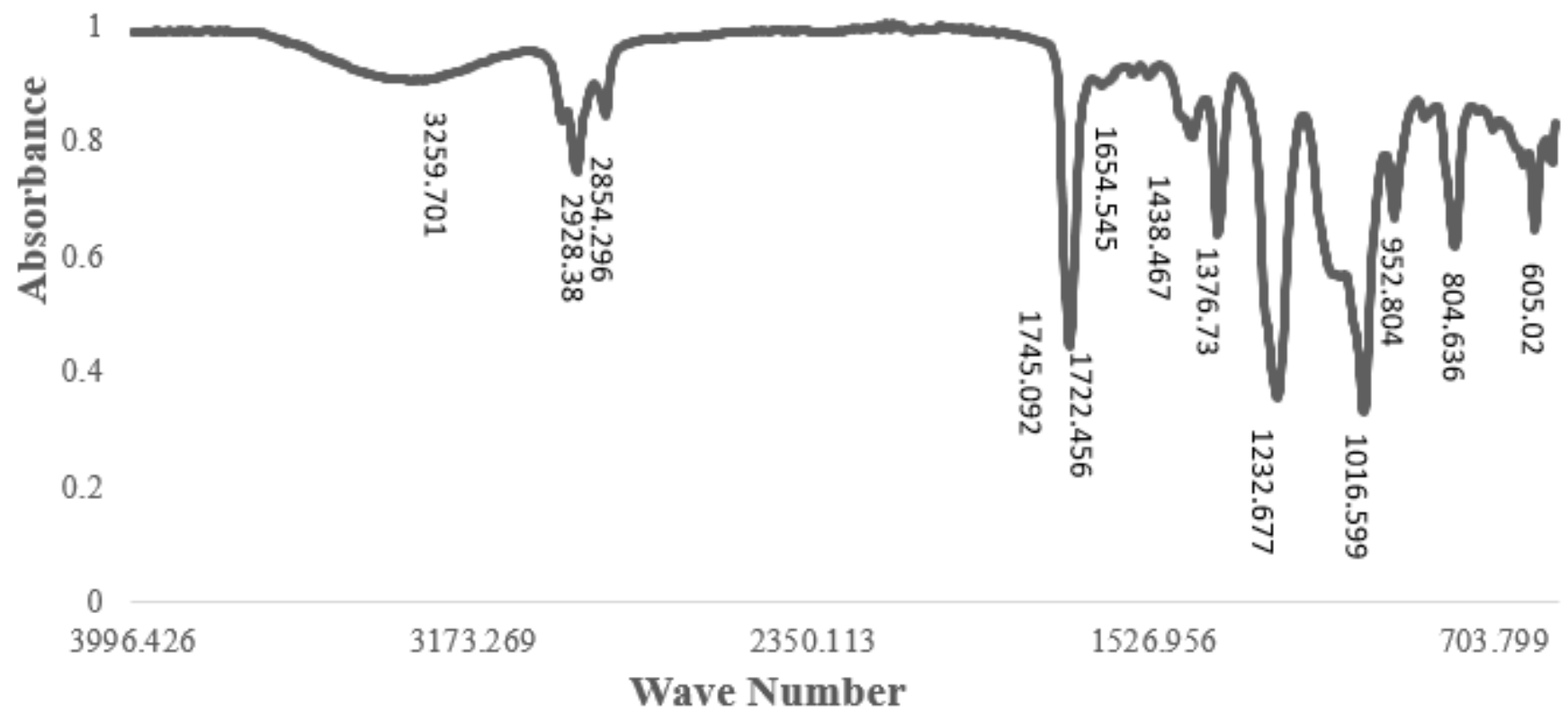


Figure 5

FT-IR Spectrum of PHA-PLA Sample of Mixed Culture

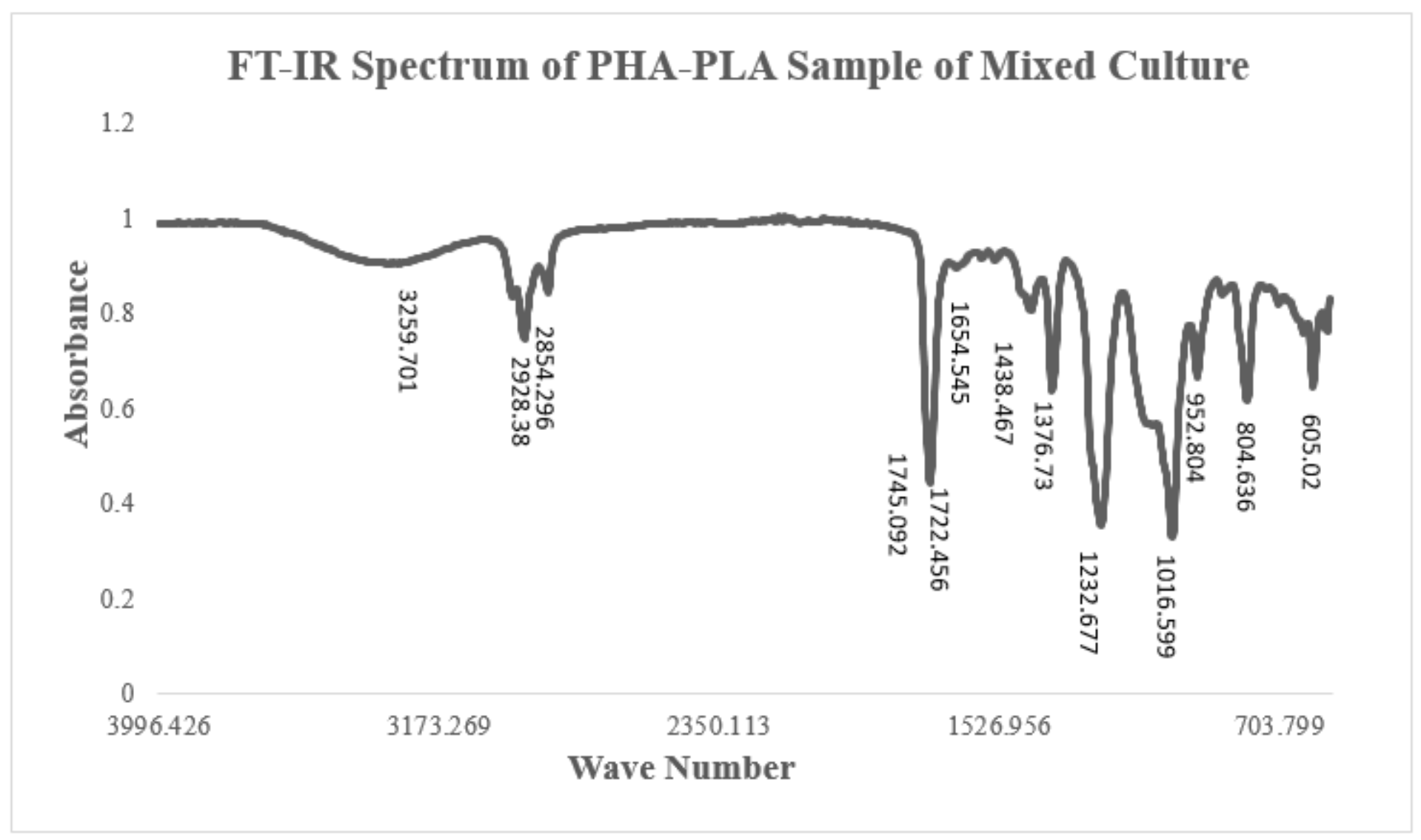

Figure 5

FT-IR Spectrum of PHA-PLA Sample of Mixed Culture 


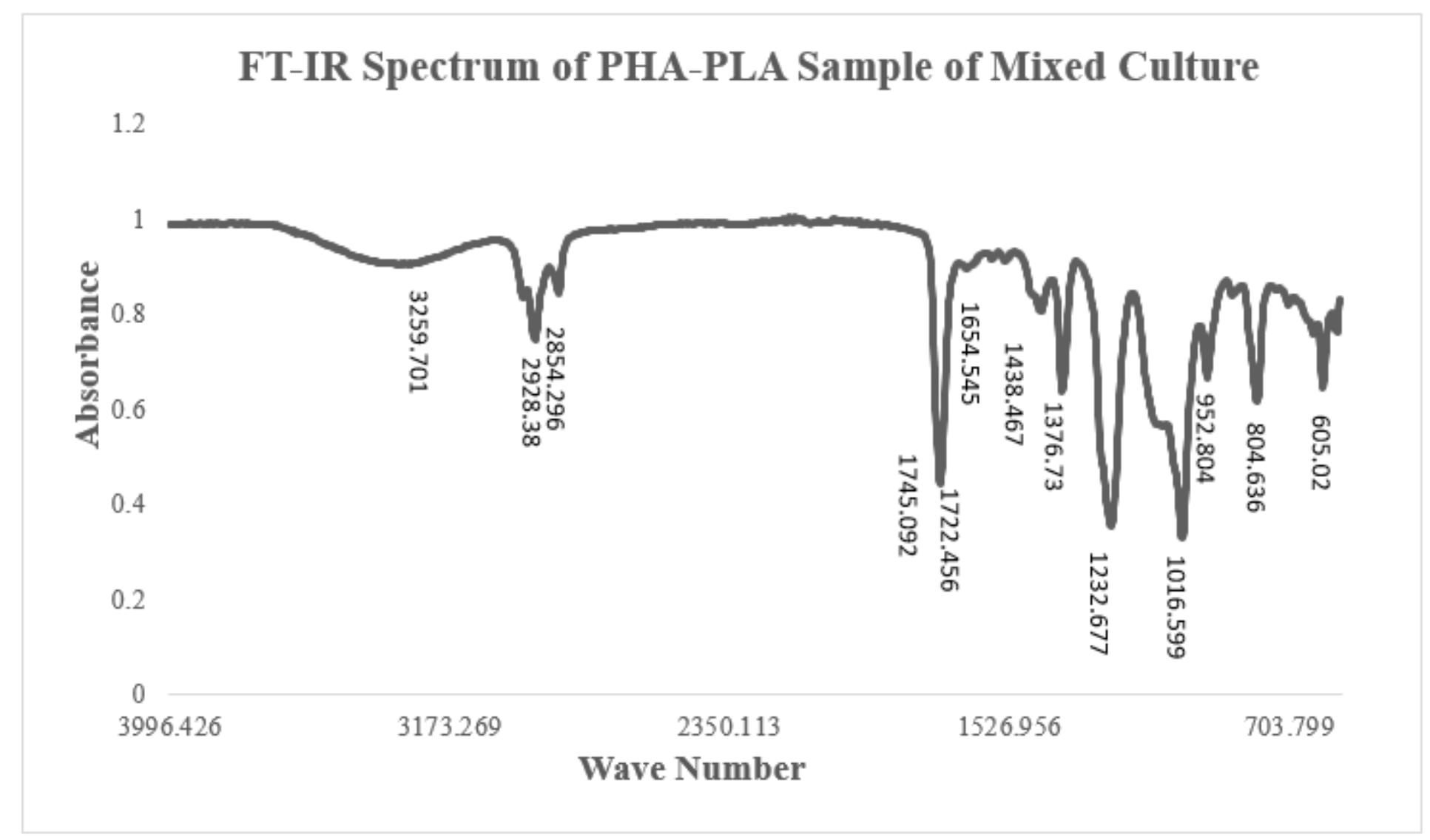

Figure 5

FT-IR Spectrum of PHA-PLA Sample of Mixed Culture 

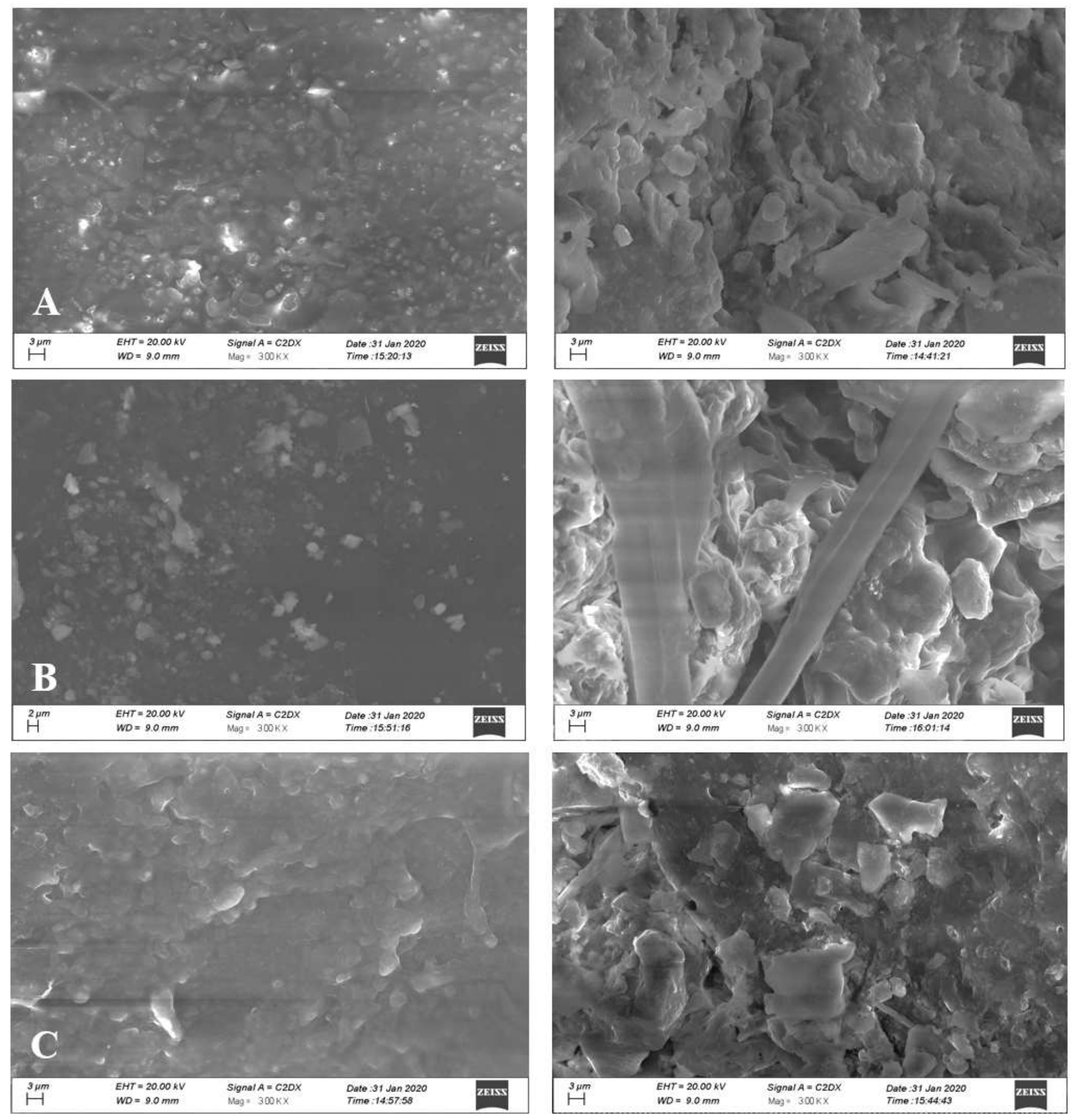

\section{Figure 6}

SEM of PHA sample F (A), PLA (B) and PHA-PLA sample PF (C) before and after degradation at $3000 \mathrm{X}$. 

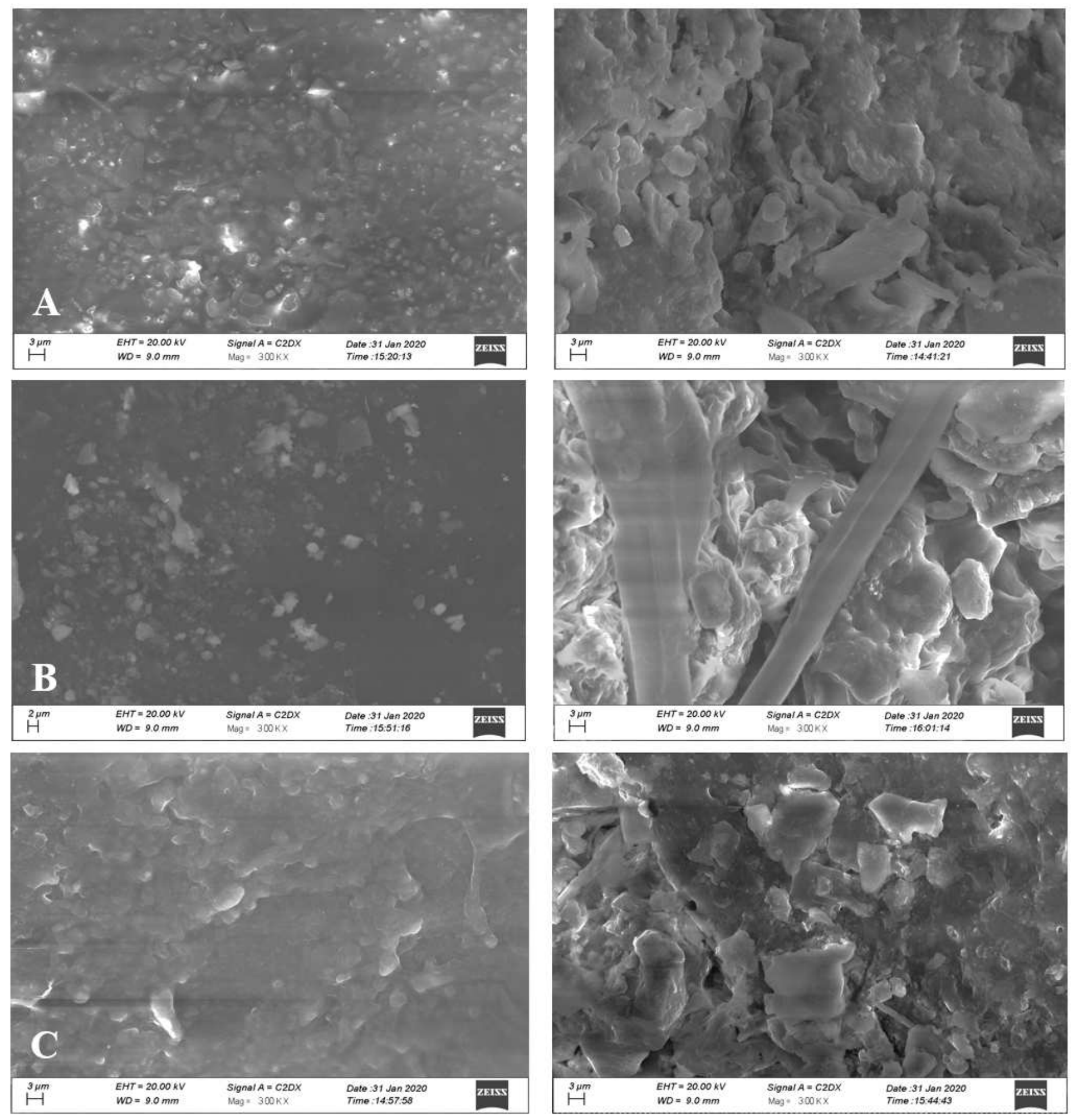

\section{Figure 6}

SEM of PHA sample F (A), PLA (B) and PHA-PLA sample PF (C) before and after degradation at $3000 \mathrm{X}$. 

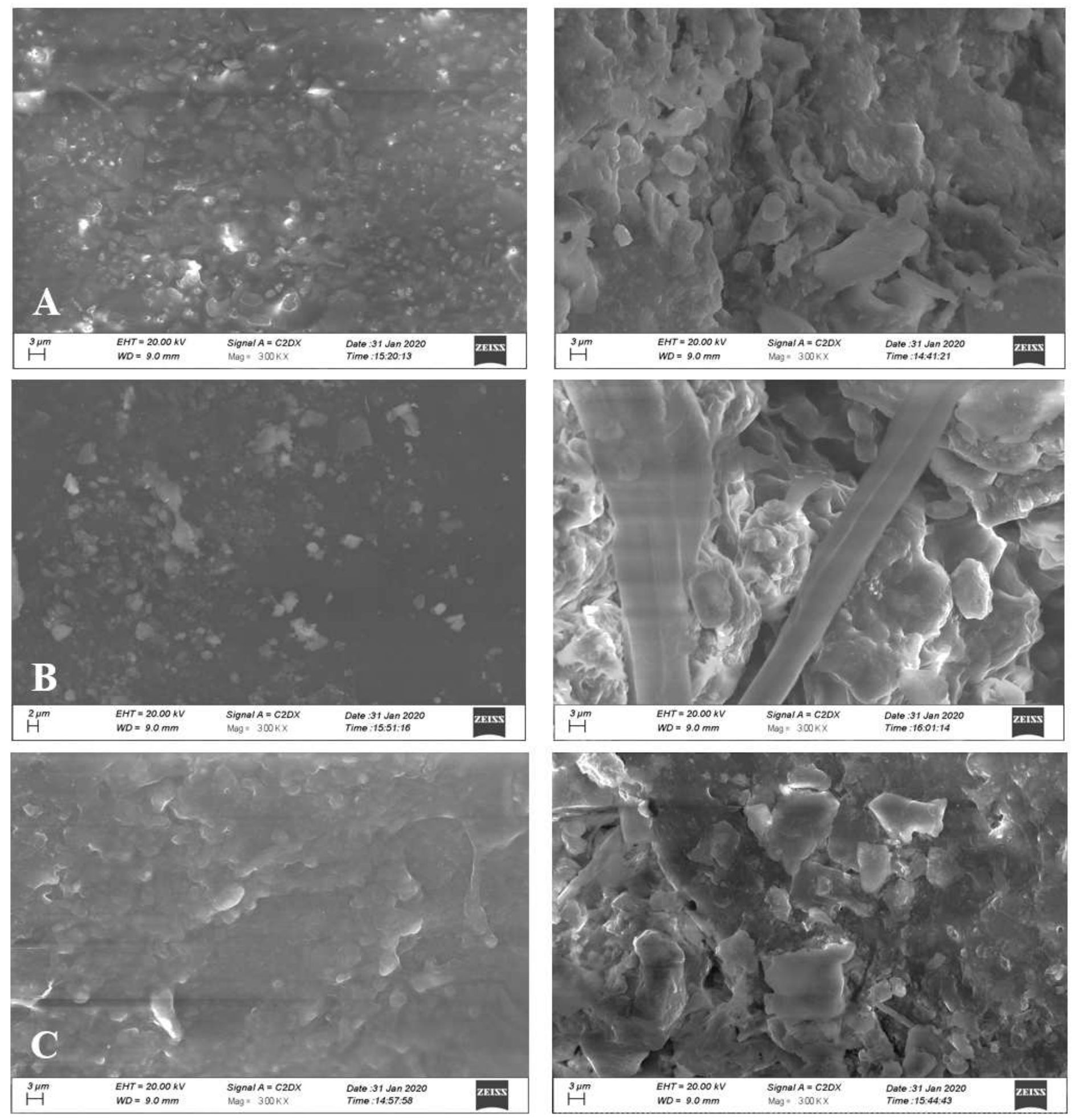

\section{Figure 6}

SEM of PHA sample F (A), PLA (B) and PHA-PLA sample PF (C) before and after degradation at $3000 \mathrm{X}$. 


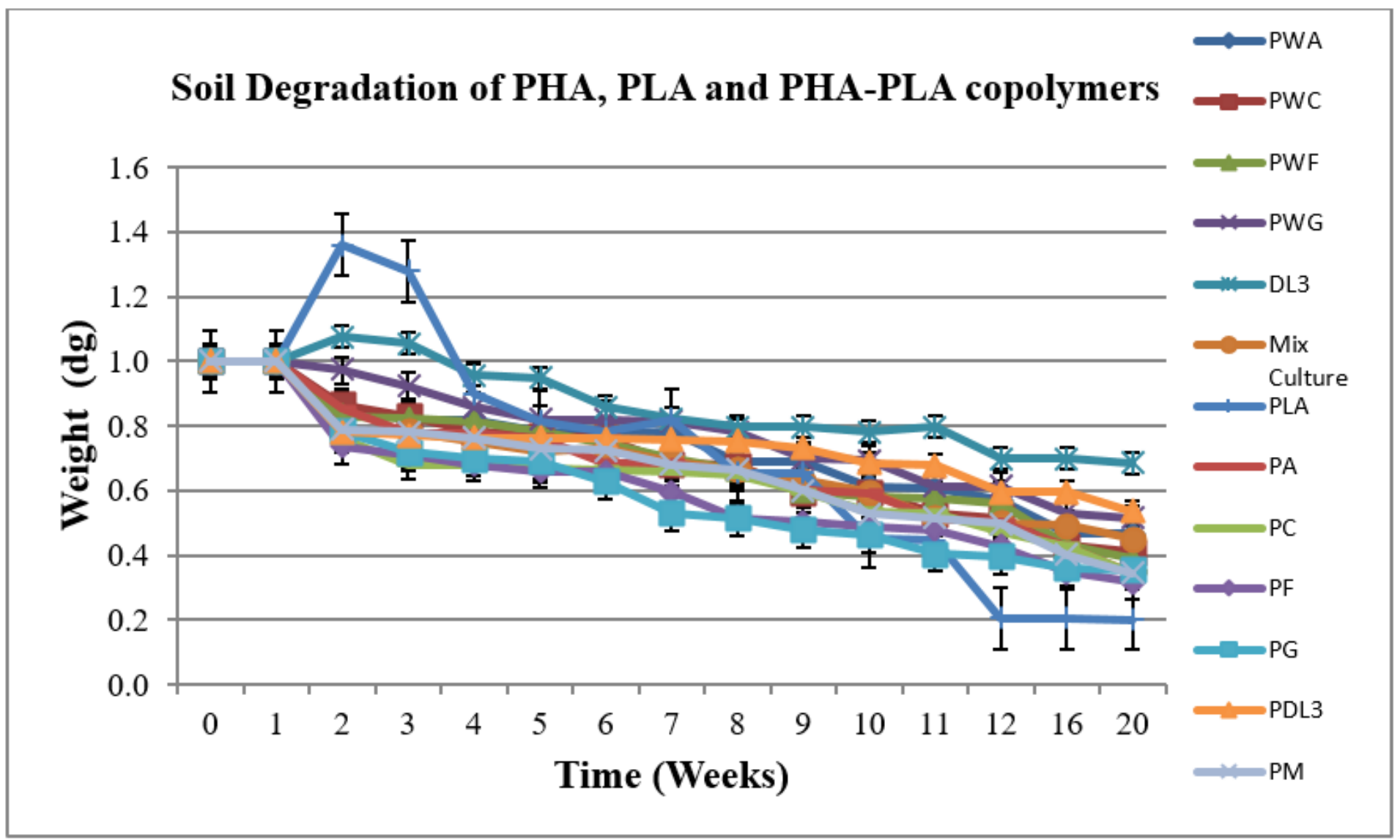

Figure 7

Graphical representation for soil degradation of PHA, PLA, and PHA-PLA blends. 


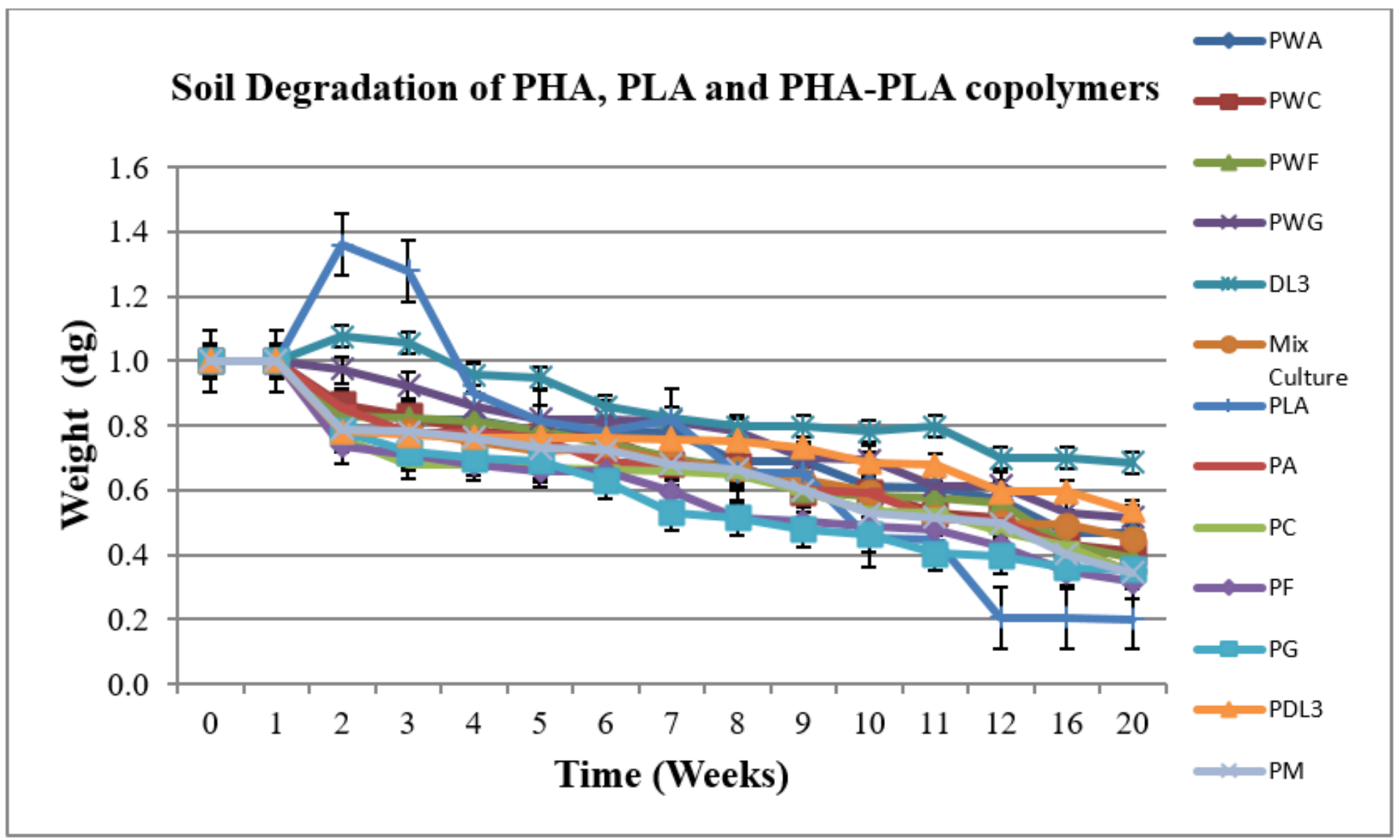

Figure 7

Graphical representation for soil degradation of PHA, PLA, and PHA-PLA blends. 


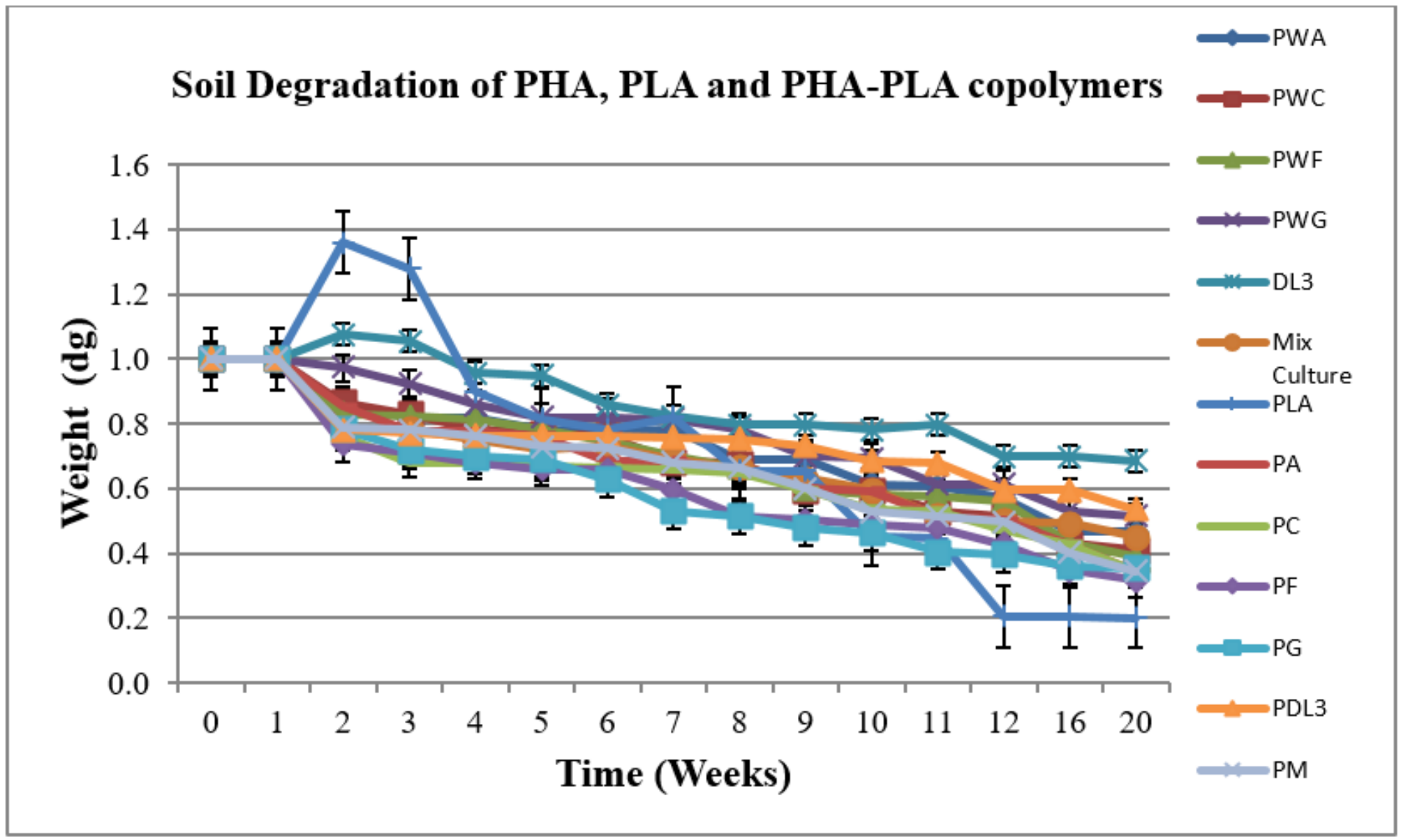

Figure 7

Graphical representation for soil degradation of PHA, PLA, and PHA-PLA blends.

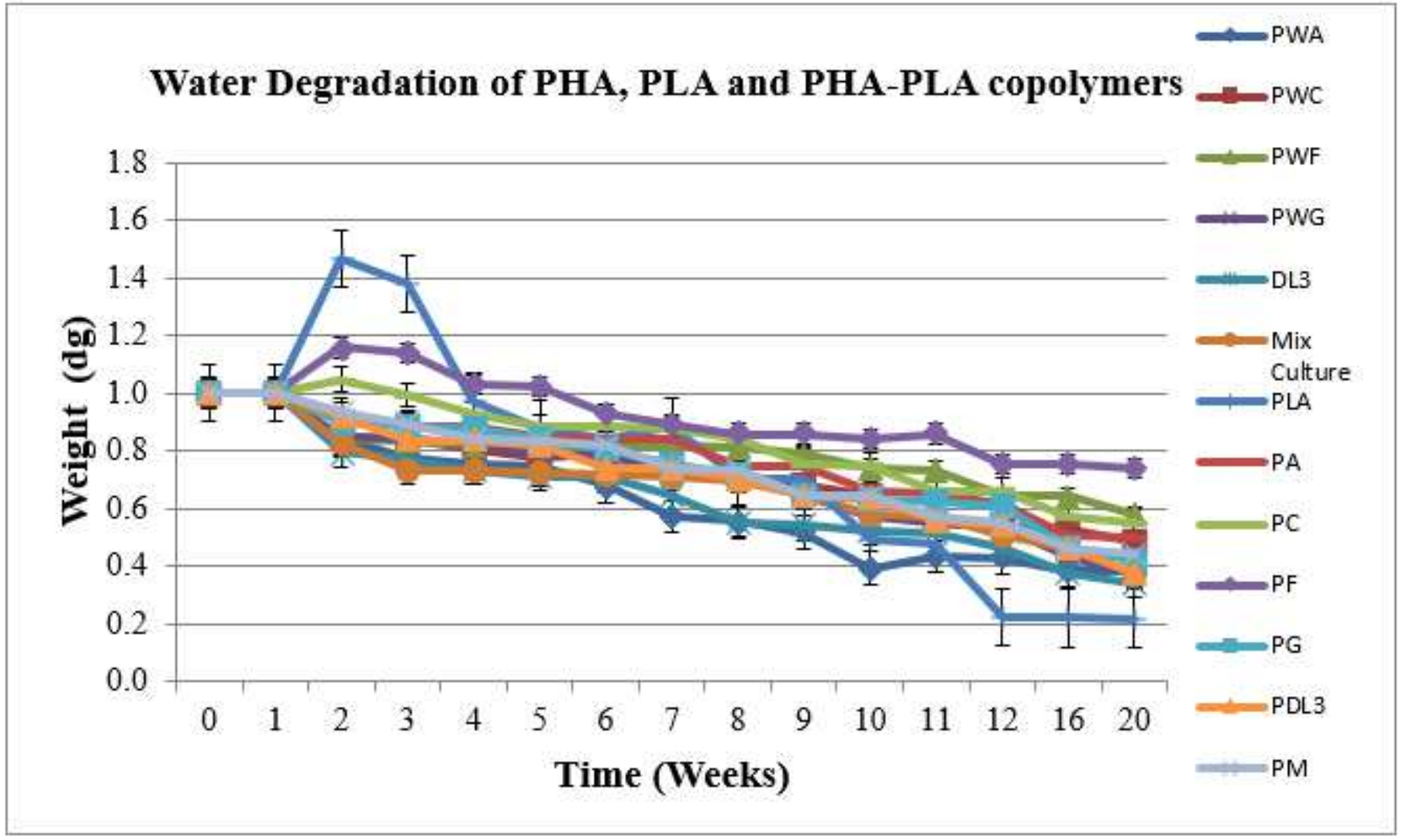


Figure 8

Graphical representation for water degradation of PHA, PLA, and PHA-PLA blends.

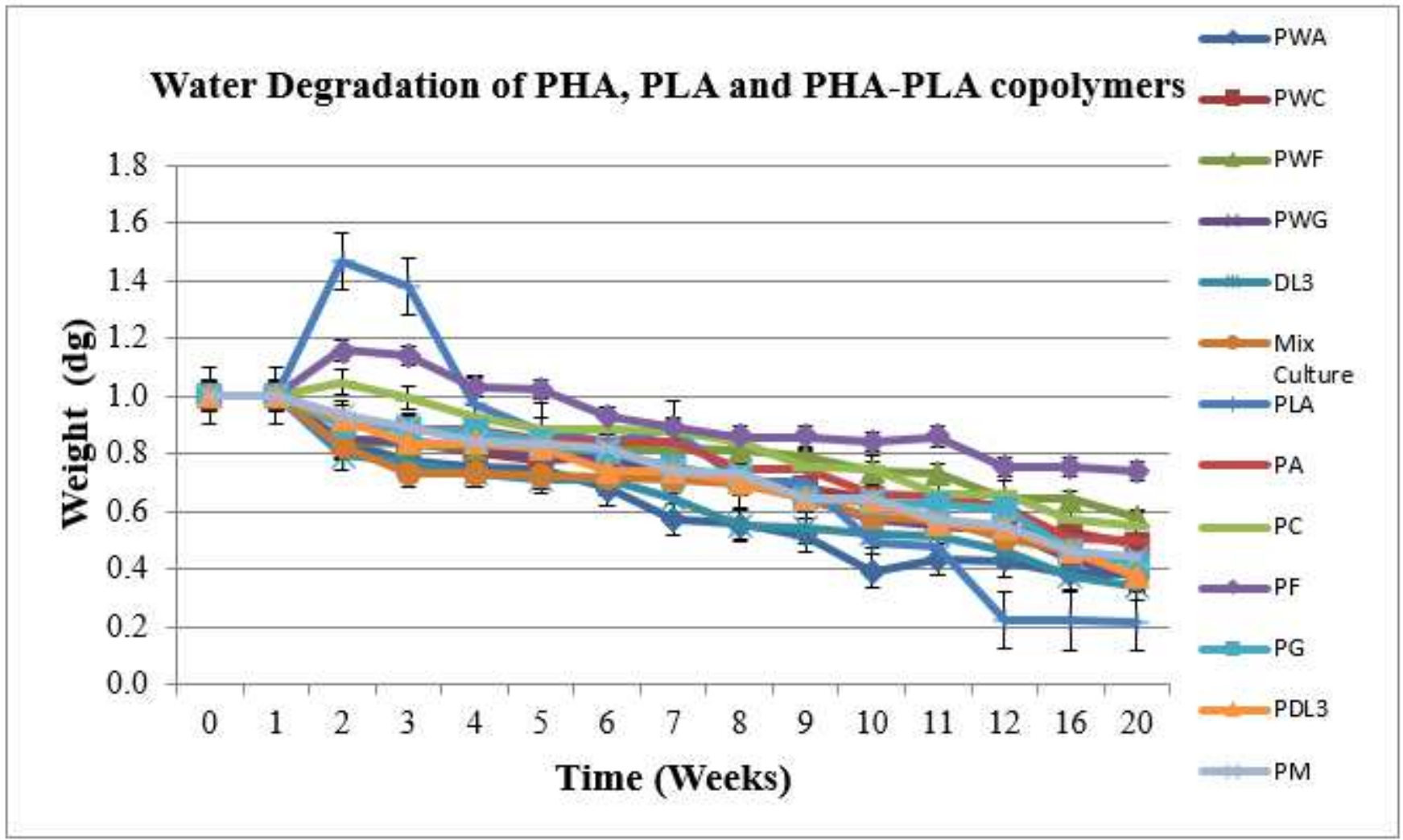

Figure 8

Graphical representation for water degradation of PHA, PLA, and PHA-PLA blends. 


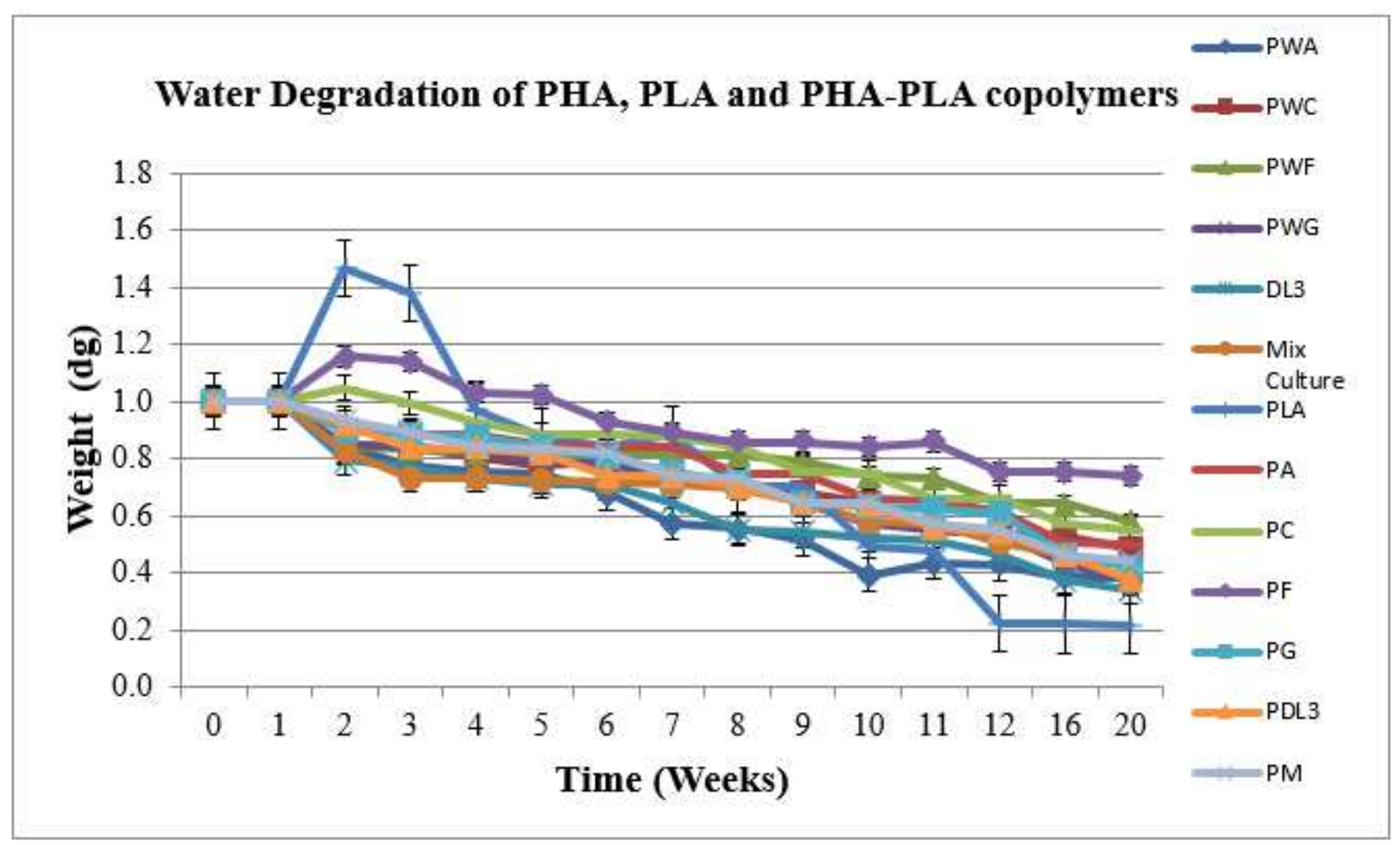

Figure 8

Graphical representation for water degradation of PHA, PLA, and PHA-PLA blends.

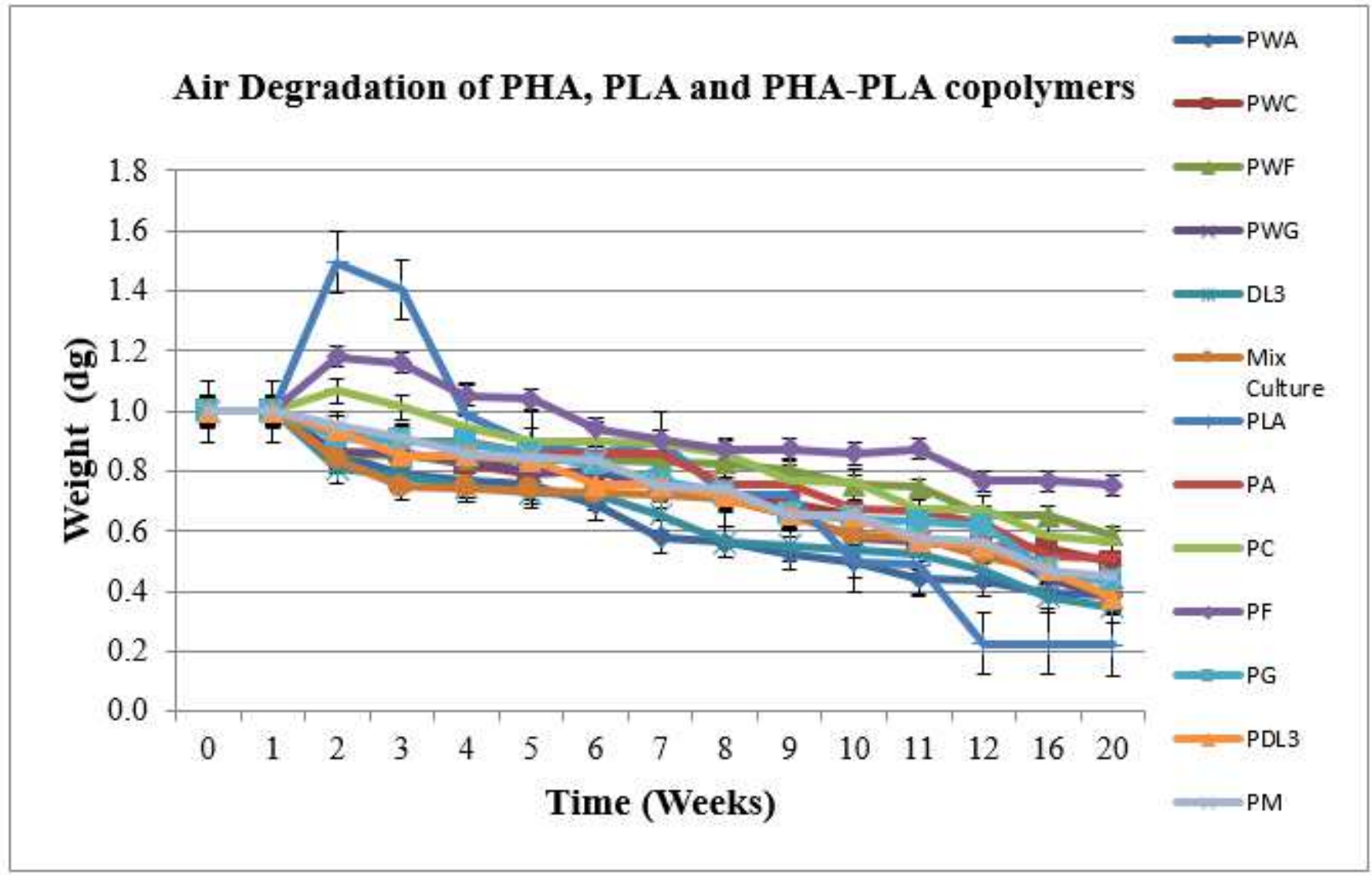


Figure 9

Graphical representation for air degradation of PHA, PLA, and PHA-PLA blends.

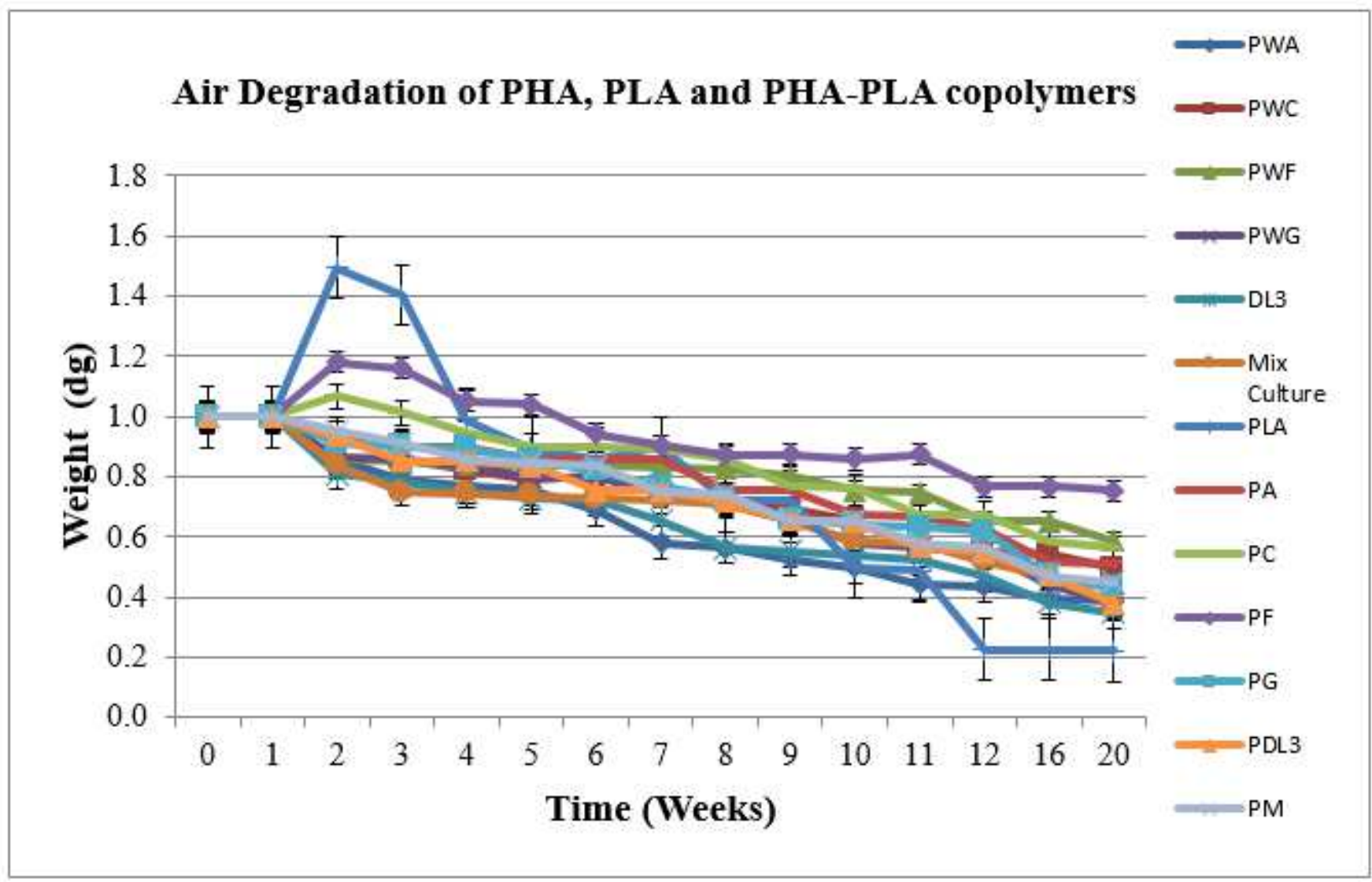

Figure 9

Graphical representation for air degradation of PHA, PLA, and PHA-PLA blends. 


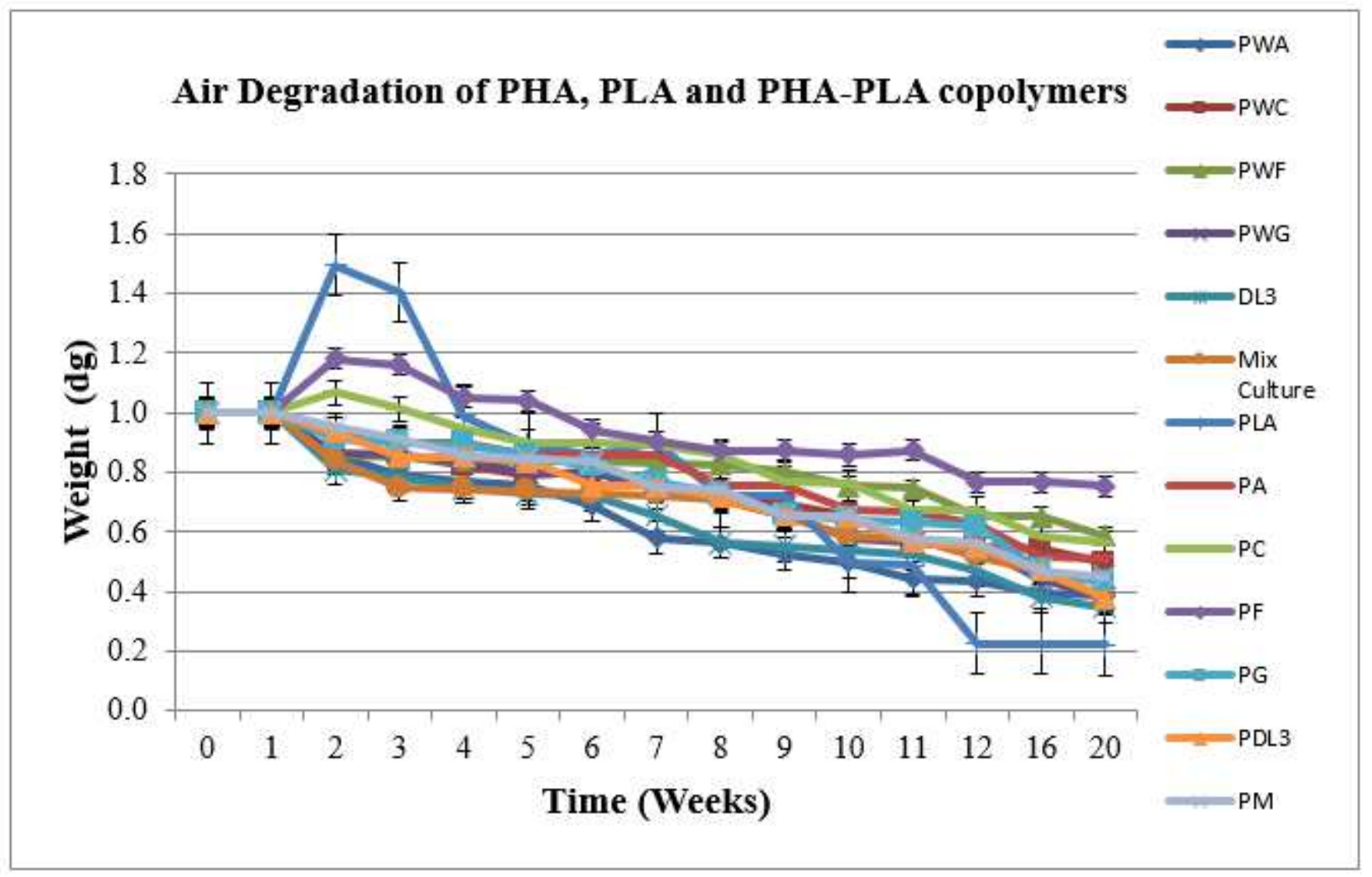

Figure 9

Graphical representation for air degradation of PHA, PLA, and PHA-PLA blends.

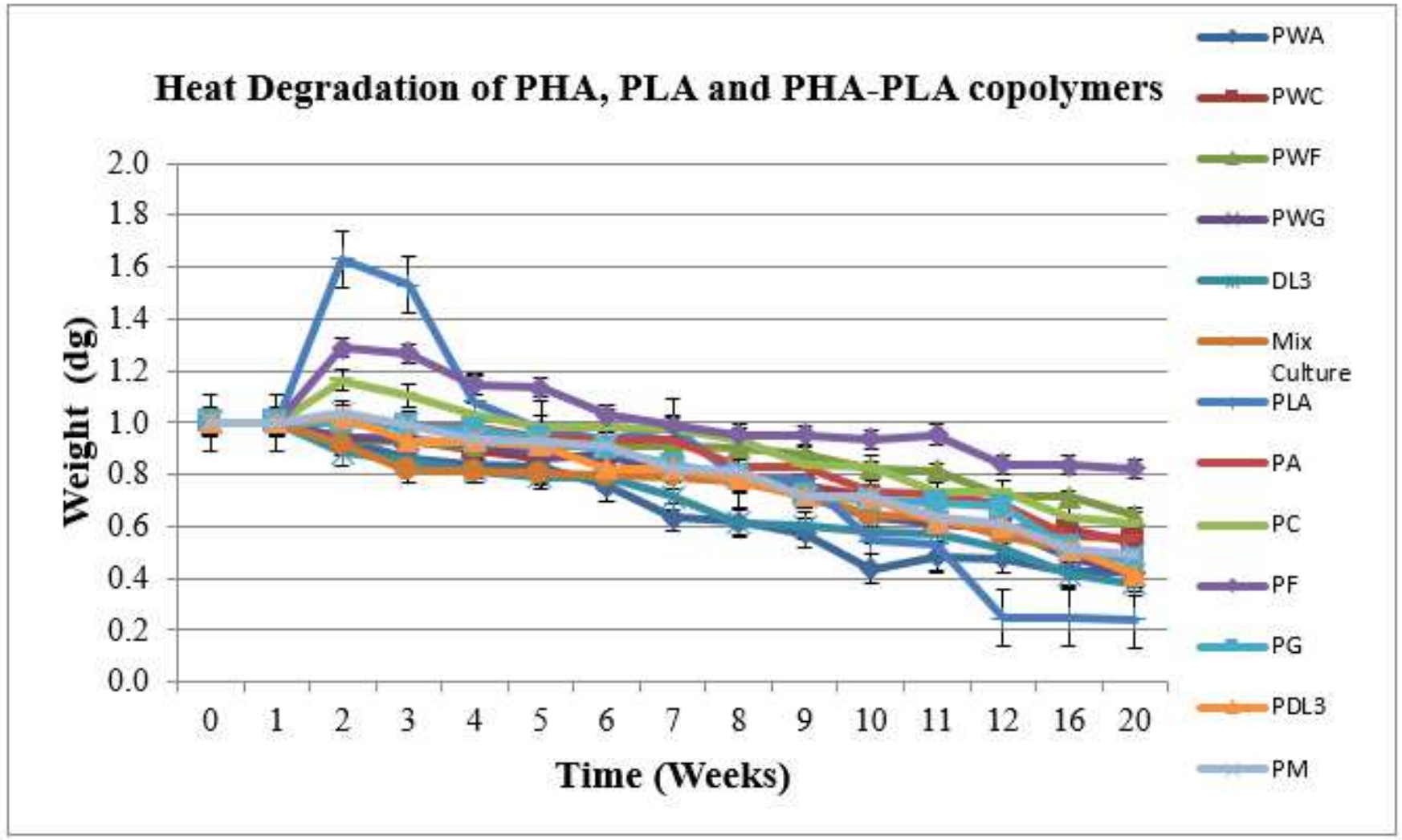


Figure 10

Graphical representation for heat degradation of PHA, PLA, and PHA-PLA blends.

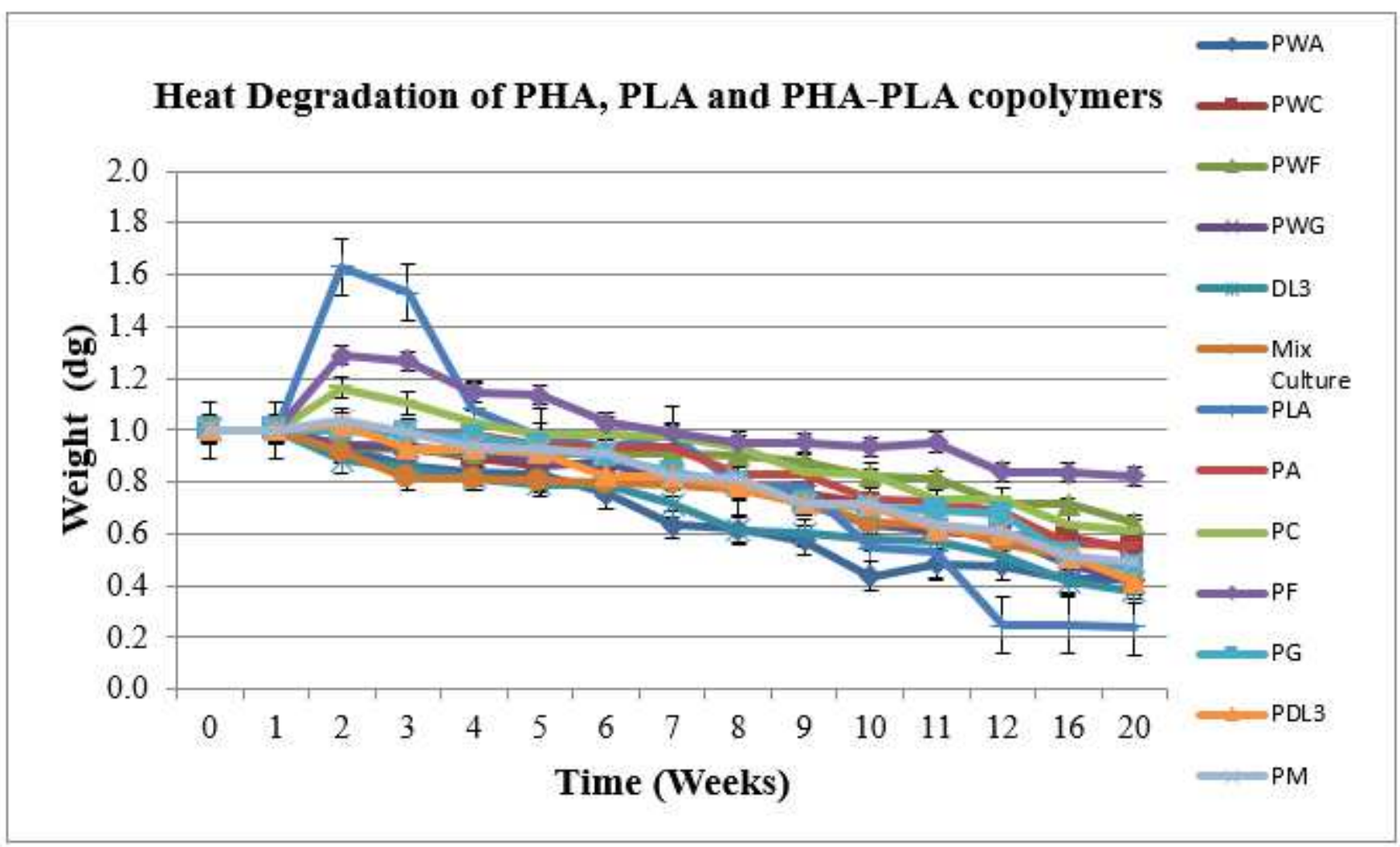

Figure 10

Graphical representation for heat degradation of PHA, PLA, and PHA-PLA blends. 


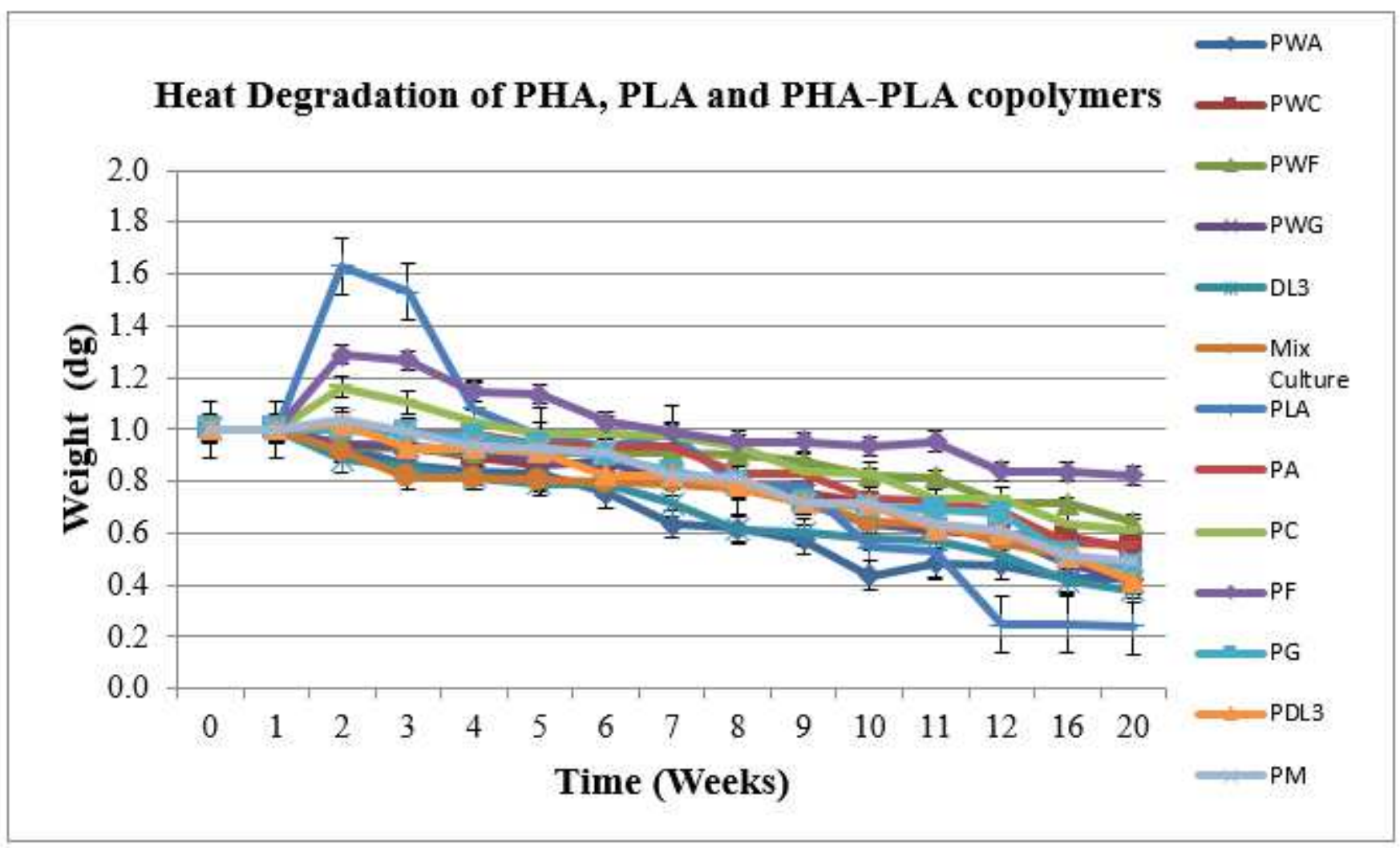

Figure 10

Graphical representation for heat degradation of PHA, PLA, and PHA-PLA blends.

\section{Supplementary Files}

This is a list of supplementary files associated with this preprint. Click to download.

- GraphicalAbstract.jpg

- GraphicalAbstract.jpg

- GraphicalAbstract.jpg 